\title{
Optimal Exercise Boundary of American Fractional Lookback Option in a Mixed Jump-Diffusion Fractional Brownian Motion Environment
}

\author{
Zhaoqiang Yang \\ Lanzhou University of Finance and Economics, Classic Library Reference Room, Lanzhou 730101, China \\ Correspondence should be addressed to Zhaoqiang Yang; woyuyanjiang@163.com
}

Received 18 April 2017; Accepted 18 May 2017; Published 17 July 2017

Academic Editor: Federica Caselli

Copyright (C) 2017 Zhaoqiang Yang. This is an open access article distributed under the Creative Commons Attribution License, which permits unrestricted use, distribution, and reproduction in any medium, provided the original work is properly cited.

\begin{abstract}
A new framework for pricing the American fractional lookback option is developed in the case where the stock price follows a mixed jump-diffusion fraction Brownian motion. By using Itô formula and Wick-Itô-Skorohod integral a new market pricing model is built. The fundamental solutions of stochastic parabolic partial differential equations are estimated under the condition of Merton assumptions. The explicit integral representation of early exercise premium and the critical exercise price are also given. Numerical simulation illustrates some notable features of American fractional lookback options.
\end{abstract}

\section{Introduction}

The mixed fractional Brownian motion $(\mathrm{mfBm})$ is a family of Gaussian processes, which is a linear combination of Brownian motion and fractional Brownian motion. The $\mathrm{mfBm}$ models have been extensively studied in the literature [1-9]. Cheridito [2] derived an European call pricing option on an asset driven by a linear combination of a Brownian motion and an independent fractional Brownian motion. Based on this theory, Sun and Yan [9] proposed a mixed fractional Brownian motion version of a credit risk pricing Merton model and assumed that the value of the firm obeys a geometric mixed fractional Brownian motion; the result shows that the mixed fractional model to simulate credit risk pricing is a reasonable one. The case even for the fractional Brownian motion with arbitrary Hurst parameter and Wick products of the fractional Black-Scholes model have been proposed as an improvement of the classical Black-Scholes model [10-12]. As is known the Black-Scholes model is inadequate to describe the asset returns and the behavior of the option markets. Chang et al. [13] proposed a jump-diffusion process with Poisson-jump to match the abnormal fluctuation of stock price. Several authors [14-18] also considered the problem of pricing options under a jumpdiffusion environment in a larger setting. Actually, various empirical studies on the statistical properties of log-returns show that the log-returns are not necessarily independent and also not Gaussian. One way to a more realistic modelling is to change the geometric Brownian motion to a geometric fractional Brownian motion: the dependence of the logreturn increments can now be modelled with the Hurst parameter of the fractional Brownian motion. It can be said that the properties of financial return series are nonnormal, nonindependent, and nonlinear, self-similar, with heavytails, in both autocorrelations and cross-correlations, and volatility clustering [2-4]. Since fractional Brownian motion has two substantial features such as self-similarity and longrange dependence, using it is more applicable to capture behavior from financial asset [3]. Also, the fractional Brownian motion is neither a Markov process nor a semimartingale, and thus we cannot apply the common stochastic calculus to analyze it. To get around this problem and to take into account the long memory property, it is reasonable to use the mixed fractional Brownian motion with jumps model to capture fluctuations of the financial asset (see [19-21]). 
Fortunately, Hu and Øksendal [12] employed the Wick product rather than the pathwise product to define a fractional stochastic integral whose mean is indeed zero. This property was very convenient for both theoretical developments and practical applications. Further, in [10], it was stated that if one uses the Wick-Itô-Skorohod integral, then they can obtain an arbitrage-free model; while Wick integration leads to no-arbitrage, the definition of the corresponding self-financing trading strategies is quite restrictive. Therefore, the fractional market based on Wick integrals is considered which is a beautiful mathematical construction but with restricted applicability in finance.

Further, to capture jumps or discontinuous, fluctuations problem or take into account long memory property, we present here a new method to solve option pricing problem for American lookback option in a mixed jump-diffusion fractional Brownian motion environment. It is different from the mixed fractional Brownian motion ones which are based on a linear combination of a Brownian motion and an independent fractional Brownian motion. We establish mixed jump-diffusion fraction Brownian motion. The mixed jump-diffusion fraction Brownian motion is a linear combination of Brownian motion, fraction Brownian motion, and Poisson process. By using Itô formula and fractional WickItô-Skorohod integral a new market pricing model is built, and the fundamental solutions of stochastic parabolic partial differential equations are estimated under the condition of Merton assumptions. Then the explicit integral representation of early exercise premium and the American fractional lookback options factorization formula are also given. Based on some researches of lookback options pricing and early exercise premium in the literature [22-28], to achieve quick and accurate pricing for practical purposes, this paper adopts the critical exercise price to value American fractional lookback options, and numerical simulation illustrates some notable features of American fractional lookback options.

The rest of this paper is organized as follows. In Section 2, we present some basic lemmas and preliminary results of mixed jump-diffusion fractional pricing model and WickItô-Skorohod integral which will be used throughout this paper. In Section 3, the fundamental solutions of stochastic parabolic partial differential equations are estimated under the condition of Merton assumptions and the optimal exercise boundary is derived; then the American fractional lookback options factorization formula is obtained. In Section 4, some simulation results and notable features are provided. The paper is ended with conclusive remarks in the last section.

\section{Mixed Jump-Diffusion Fractional Brownian Motion Pricing Model and Wick-Itô-Skorohod Integral}

In this section we construct mixed Poisson-jump-diffusion processes as a suitable alternative to fractional Brownian motion.
Definition 1 (see $[1,3,5]$ ). A mixed fractional Brownian motion of parameters $\alpha, \beta$, and $H$ is a linear combination of fractional Brownian motion ( $\mathrm{fBm}$ ) with Hurst parameter $H$ and Brownian motion, defined on the probability space $(\Omega, \mathfrak{\mho}, \mathbb{P})$ for any $t \in \mathbb{R}^{+}$by

$$
M_{t}^{H}=\alpha B_{t}+\beta B_{t}^{H},
$$

where $B_{t}$ is a Brownian motion, $B_{t}^{H}$ is an independent $\mathrm{fBM}$ with $H \in(0,1)$, and $\alpha, \beta$ are two real constants such that $(\alpha, \beta) \neq(0,0)$.

Now we list some properties in [1] by the following proposition.

Proposition 2. The $m f B m M_{t}^{H}$ satisfies the following properties:

(i) $M_{t}^{H}$ is a centered Gaussian process and not a Markovian one for all $H \in(0,1) \backslash 1 / 2$;

(ii) $M_{0}^{H}=0 \mathbb{P}$-almost surely;

(iii) the covariation function of $M_{t}^{H}(\alpha, \beta)$ and $M_{s}^{H}(a, b)$ for any $t, s \in \mathbb{R}^{+}$is given by

$$
\begin{aligned}
\operatorname{Cov}\left(M_{t}^{H}, M_{s}^{H}\right)= & \alpha^{2}(t \wedge s) \\
& +\frac{\beta^{2}}{2}\left(t^{2 H}+s^{2 H}-|t-s|^{2 H}\right),
\end{aligned}
$$

where $\wedge$ denotes the minimum of two numbers;

(iv) the increments of $M_{t}^{H}(\alpha, \beta)$ are stationary and mixedself-similar for any $h>0$

$$
M_{h t}^{H}(\alpha, \beta) \triangleq M_{t}^{H}\left(\alpha h^{1 / 2}, \beta h^{H}\right),
$$

where $\triangleq$ means "to have the same law";

(v) the increments of $M_{t}^{H}$ are positively correlated if $1 / 2<$ $H<1$, uncorrelated if $H=1 / 2$, and negatively correlated if $0<H<1 / 2$;

(vi) the increments of $M_{t}^{H}$ are long-range dependent if, and only if, $H>1 / 2$;

(vii) for all $t \in \mathbb{R}^{+}$, we have

$$
\begin{aligned}
E[ & \left.\left(M_{t}^{H}(\alpha, \beta)\right)^{n}\right] \\
& = \begin{cases}0, & n=2 l+1 \\
\frac{(2 l) !}{2^{l} l !}\left(\alpha^{2} t+\beta^{2} t^{2 H}\right)^{l}, & n=2 l .\end{cases}
\end{aligned}
$$

Proof. These properties are easily obtained based on $[1$, 2].

Now, let $(\Omega, \mathfrak{\Im}, \mathbb{P})$ be a probability field such that $B_{t}$ is a Brownian motion with respect to $\mathbb{P}$ and $B_{t}^{H}$ is an independent fractional Brownian motion with respect to $\mathbb{P}$. Some results presented that is needed for the following Lemma $[2,3,5]$. 
Lemma 3. For every $0<t<T$ and $\sigma \in \mathbb{C}$ one has

$$
\widetilde{E}_{t}\left[\mathrm{e}^{\sigma\left(B_{T}+B_{T}^{H}\right)}\right]=\mathrm{e}^{\sigma\left(B_{T}+B_{T}^{H}\right)+(1 / 2)(T-t) \sigma^{2}+(1 / 2) \sigma^{2}\left(T^{2 H}-t^{2 H}\right)},
$$

where $\widetilde{E}_{t}$ denotes the quasi-conditional expectation with respect to the risk-neutral measure.

Lemma 4. Let $f$ be a function such that $\widetilde{E}_{t}\left[f\left(B_{T}, B_{T}^{H}\right)\right]<\infty$. Then for every $0<t \leq T$ and $\sigma \in \mathbb{C}$,

$$
\widetilde{E}_{t}\left[f\left(\sigma B_{T}+\sigma B_{T}^{H}\right)\right]=\int_{\mathbb{R}} \frac{1}{\sqrt{2 \pi\left[\sigma^{2}\left(T-t+T^{2 H}-t^{2 H}\right)\right]}} \exp \left[-\frac{\left(x-\sigma B_{t}-\sigma B_{t}^{H}\right)^{2}}{2 \sigma^{2}\left(T-t+T^{2 H}-t^{2 H}\right)}\right] \cdot f(x) \mathrm{d} x .
$$

Lemma 5. Let $A \in \mathfrak{B}(\mathbb{R}), f(x)=\mathbf{1}_{A}(x)$. Then

$$
\tilde{E}_{t}\left[\mathbf{1}_{A}\left(\sigma B_{T}+\sigma B_{T}^{H}\right)\right]=\int_{\mathbb{R}} \frac{1}{\sqrt{2 \pi\left[\sigma^{2}\left(T-t+T^{2 H}-t^{2 H}\right)\right]}} \exp \left[-\frac{\left(x-\sigma B_{t}-\sigma B_{t}^{H}\right)^{2}}{2 \sigma^{2}\left(T-t+T^{2 H}-t^{2 H}\right)}\right] \mathbf{1}_{A}(x) \mathrm{d} x .
$$

Let $\sigma_{1}, \sigma_{2} \in \mathbb{R}$. Consider the process

$$
\begin{array}{r}
\mathbb{Z}_{t}^{*}=\sigma_{1} B_{t}^{*}+\sigma_{2}\left(B_{t}^{H}\right)^{*}=\sigma_{1} B_{t}+\sigma_{1}^{2} t+\sigma_{2} B_{t}^{H}+\sigma_{2}^{2} B_{t}^{2 H}, \\
0 \leq t \leq T,
\end{array}
$$

where $\sigma_{1}$ and $\sigma_{2}$ are two real constants such that $\left(\sigma_{1}, \sigma_{2}\right) \neq$ $(0,0)$. From Girsanov's theorem, there exists a measure $\mathbb{P}^{*}$, such that $\mathbb{Z}_{t}^{*}$ is a new $m f B m$. We denote by $E_{t}^{*}[\cdot]$ the quasiconditional expectation with respect to $\mathbb{P}^{*}$ as follows:

$$
\begin{aligned}
E_{t}^{*} & {\left[\mathrm{e}^{\sigma_{1} B_{T}+\sigma_{2}\left(B_{T}^{H}\right)^{*}}\right] } \\
& =\mathrm{e}^{\sigma_{1}\left(B_{T}+B_{T}^{H}\right)+(1 / 2) \sigma_{1}^{2}(T-t)+(1 / 2) \sigma_{2}^{2}\left(T^{2 H}-t^{2 H}\right)} .
\end{aligned}
$$

Lemma 6. Let $f$ be a function such that $\widetilde{E}_{t}\left[f\left(\sigma_{1} B_{T}+\sigma_{2} B_{T}^{H}\right)\right]<$ $\infty$. Let $X_{t}=\exp \left(-\sigma_{1} B_{t}-\left(\sigma_{1}^{2} / 2\right) t-\sigma_{2} B_{t}^{H}-\left(\sigma_{2}^{2} / 2\right) t^{2 H}\right)$. Then for every $t \leq T$, we have

$$
\begin{aligned}
\widetilde{E}_{t}^{*} & {\left[f\left(\sigma_{1} B_{T}+\sigma_{2} B_{T}^{H}\right)\right] } \\
& =\frac{1}{X_{t}} \widetilde{E}_{t}\left[f\left(\sigma_{1} B_{T}+\sigma_{2} B_{T}^{H}\right) X_{T}\right] .
\end{aligned}
$$

Lemma 7. The price at every $t \in[0, T]$ of a bounded $F_{T}^{H}$ measurable claim $F \in L^{2}$ is given by

$$
F_{t}=\mathrm{e}^{-r(T-t)} \widetilde{E}_{t}[F]
$$

where represents the constant riskless interest rate.

The proof of Lemmas 3-7 can be seen in $[2,3,5]$.

2.1. Mixed Jump-Diffusion Fractional Brownian Motion Pricing Model. Suppose an option whose value $V_{t}=V\left(S_{t}, t\right)$ depends only on $S_{t}$, $t$, and option pricing formula while the dynamics of stock $\log$-price $S_{t}$ satisfies

$$
\mathrm{d} S_{t}=\mu S_{t} \mathrm{~d} t+\sigma_{1} S_{t} \mathrm{~d} B_{t}^{H}+\sigma_{2} S_{t} \mathrm{~d} B_{t},
$$

where $\mu$ is the instantaneous expected return, $\sigma_{1}$ and $\sigma_{2}$ are the instantaneous volatility, $B_{t}$ is a standard Brownian motion, and $B_{t}^{H}$ is a fractional Brownian motion. Assume that $B_{t}$ and $B_{t}^{H}$ are independent.

Lemma 8. Let $V_{t}=V\left(S_{t}, t\right)$ be a binary differential function; if stochastic process $S_{t}$ is suitable for stochastic differential equation (12), then

$$
\begin{aligned}
\mathrm{d} V_{t} & =\left(\frac{\partial V}{\partial t}+\mu S_{t} \frac{\partial V}{\partial S}+H \sigma_{1}^{2} S_{t}^{2} t^{2 H-1} \frac{\partial^{2} V}{\partial S^{2}}\right. \\
& \left.+\frac{1}{2} \sigma_{2}^{2} S_{t}^{2} \frac{\partial^{2} V}{\partial S^{2}}\right) \mathrm{d} t+\sigma_{1} S_{t} \frac{\partial V}{\partial S} \mathrm{~d} B_{t}^{H}+\sigma_{2} S_{t} \frac{\partial V}{\partial S} \mathrm{~d} B_{t}
\end{aligned}
$$

Proof. According to Taylor expansion we have

$$
\mathrm{d} V_{t}=\frac{\partial V}{\partial t} \mathrm{~d} t+\frac{\partial V}{\partial S} \mathrm{~d} S_{t}+\frac{1}{2} \frac{\partial^{2} V}{\partial S^{2}}\left(\mathrm{~d} S_{t}\right)^{2}+o\left(\mathrm{~d} t \mathrm{~d} S_{t}\right)
$$

Notice that $\mathbb{C}^{H}(t, s)=(1 / 2)\left\{|t|^{2 H}+|s|^{2 H}-|t-s|^{2 H}\right\}$; then $\mathbb{E}\left[B_{t}^{H}\right]^{2}=t^{2 H}, \mathbb{E}\left[B_{t}\right]^{2}=t$; since we can approximate that $\left(\mathrm{d} B_{t}^{H}\right)^{2}=2 H t^{2 H-1} \mathrm{~d} t,\left(\mathrm{~d} B_{t}\right)^{2}=\mathrm{d} t,(\mathrm{~d} t)^{2}=0, \mathrm{~d} t \mathrm{~d} B_{t}=0$, we get

$$
\begin{aligned}
& \left(\mathrm{d} S_{t}\right)^{2}=\left[\mu S_{t} \mathrm{~d} t+\sigma_{1} S_{t} \mathrm{~d} B_{t}^{H}+\sigma_{2} S_{t} \mathrm{~d} B_{t}\right]^{2}=\mu^{2} S_{t}^{2}(\mathrm{~d} t)^{2} \\
& \quad+\sigma_{1}^{2} S_{t}^{2}\left(\mathrm{~d} B_{t}^{H}\right)^{2}+\sigma_{2}^{2} S_{t}^{2}\left(\mathrm{~d} B_{t}\right)^{2} \\
& \quad+2\left[\mu \sigma_{1} S_{t}^{2} \mathrm{~d} B_{t}^{H} \mathrm{~d} t+\mu \sigma_{2} S_{t}^{2} \mathrm{~d} B_{t} \mathrm{~d} t+\sigma_{1} \sigma_{2} S_{t}^{2} \mathrm{~d} B_{t}^{H} \mathrm{~d} B_{t}\right] \\
& \quad=2 H \sigma_{1}^{2} S_{t}^{2} t^{2 H-1} \mathrm{~d} t+\sigma_{2}^{2} S_{t}^{2} \mathrm{~d} t+o(\mathrm{~d} t) .
\end{aligned}
$$


Combining (14) with (15) we get

$$
\begin{aligned}
& \mathrm{d} V_{t}=\frac{\partial V}{\partial t} \mathrm{~d} t+\frac{\partial V}{\partial S}\left[\mu S_{t} \mathrm{~d} t+\sigma_{1} S_{t} \mathrm{~d} B_{t}^{H}+\sigma_{2} S_{t} \mathrm{~d} B_{t}\right]+\frac{1}{2} \\
& \frac{\partial^{2} V}{\partial S^{2}}\left[2 H \sigma_{1}^{2} S_{t}^{2} t^{2 H-1} \mathrm{~d} t+\sigma_{2}^{2} S_{t}^{2} \mathrm{~d} t\right]=\left(\frac{\partial V}{\partial t}\right. \\
&\left.+\mu S_{t} \frac{\partial V}{\partial S}+H \sigma_{1}^{2} S_{t}^{2} t^{2 H-1} \frac{\partial^{2} V}{\partial S^{2}}+\frac{1}{2} \sigma_{2}^{2} S_{t}^{2} \frac{\partial^{2} V}{\partial S^{2}}\right) \mathrm{d} t \\
&+\sigma_{1} S_{t} \frac{\partial V}{\partial S} \mathrm{~d} B_{t}^{H}+\sigma_{2} S_{t} \frac{\partial V}{\partial S} \mathrm{~d} B_{t} .
\end{aligned}
$$

Consider a continuous-time financial market in $[0, T]$. It can be described by a filtered complete probability space $\left\{\Omega, \mathfrak{F}, \mathfrak{F}_{t}, \mathbb{P}\right\} .\left\{\mathfrak{F}_{t}\right\}_{0 \leq t \leq T} \equiv \mathbb{F}$ is a natural $\sigma$-filtration generated by a standard Brownian motion $B_{t}$, fractional Brownian motion $B_{t}^{H}$, and a Poisson process $P_{t}$. Here $P_{t}$ is an $\left(\mathfrak{F}_{t}, \mathbb{P}\right)$ Poisson-jump process with intensity $\lambda$, independent of $B_{t}$ and $B_{t}^{H} \cdot j_{t}$ is jump percent at time $t$ and i.i.d.; $j_{t}$ satisfies Merton assumptions $\ln \left(1+j_{t}\right) \sim \mathbb{N}\left[\ln \left(1+\mu_{j}\right)-(1 / 2) \sigma_{j}^{2}, \sigma_{j}^{2}\right]$, where $\mu_{j}$ is the unconditional expectation of $j_{t}$, and $\sigma_{j}^{2}$ is the variance of $\ln \left(1+j_{t}\right)$. Notice that the unconditional expectation $\mu_{j}$ is deterministic and can be calculated; $j_{t}$ is a bounded function of $t$, so we assume that $\sigma_{3}=\ln \left(1+\mu_{j}\right)$ is a constant. By Lemma 8 we have Lemma 9 as follows.

Lemma 9. Let $V_{t}=V\left(S_{t}, t\right)$ be a binary differential function; if stochastic process $S_{t}$ is suitable for stochastic differential equation

$$
\mathrm{d} S_{t}=\mu S_{t} \mathrm{~d} t+\sigma_{1} S_{t} \mathrm{~d} B_{t}^{H}+\sigma_{2} S_{t} \mathrm{~d} B_{t}+\sigma_{3} S_{t} \mathrm{~d} P_{t},
$$

then

$$
\begin{aligned}
\mathrm{d} V_{t} & =\left[\frac{\partial V}{\partial t}+\mu S_{t} \frac{\partial V}{\partial S}\right. \\
& \left.+\left(H \sigma_{1}^{2} S_{t}^{2} t^{2 H-1}+\frac{1}{2} \sigma_{2}^{2} S_{t}^{2}+\frac{1}{2} \lambda \sigma_{3}^{2} S_{t}^{2}\right) \frac{\partial^{2} V}{\partial S^{2}}\right] \mathrm{d} t \\
& +\sigma_{1} S_{t} \frac{\partial V}{\partial S} \mathrm{~d} B_{t}^{H}+\sigma_{2} S_{t} \frac{\partial V}{\partial S} \mathrm{~d} B_{t}+\sigma_{3} S_{t} \frac{\partial V}{\partial S} \mathrm{~d} P_{t} \\
& +\lambda \mathbb{E}\left[V\left(S\left(1+j_{t}\right), t\right)-V(S, t)\right] \mathrm{d} t,
\end{aligned}
$$

where $\sigma_{3} S_{t}(\partial V / \partial S) \mathrm{d} P_{t}$ is the change volume of Poisson-jump process within $\mathrm{d} t$ for $\partial V / \partial S, \lambda \mathbb{E}\left[V\left(S\left(1+j_{t}\right), t\right)-V(S, t)\right] \mathrm{d} t$ is the change volume of Poisson-jump process within $\mathrm{d} t$, and $\mathbb{E}$ is the expectation operator of $V$.

Proof. Let $\mathrm{d} P_{t}$ admit two-point distribution as follows:

$$
\mathrm{d} P_{t}= \begin{cases}P\left\{j_{t}=\omega_{1}\right\}=p, & \text { jumps can not occur } \\ P\left\{j_{t}=\omega_{2}\right\}=1-p, & \text { jumps can occur. }\end{cases}
$$

Moreover, during the time interval $[t, t+\mathrm{d} t]$, we can write the probability that jumps can not occur as $\operatorname{Prob}\left(\omega_{1}\right)=1-\lambda \mathrm{d} t$ and the probability that jumps can occur as $\operatorname{Prob}\left(\omega_{2}\right)=\lambda \mathrm{d} t$.
In the case $j_{t}>0, S_{t+}>S_{t}$ describes stock price $S_{t}$ has upward jump at time $t$. In the case $j_{t}>0$, while $S_{t+}<S_{t}$ describes stock price $S_{t}$ has downward jump at time $t$. Hence $j_{t}>-1$ can ensure the stock price is positive, such that $S_{t+}=S_{t}(1+$ $\left.j_{t}\right)>0$.

Let $\Pi_{t}=V_{t}-\nabla_{t} S_{t}$ be a riskless portfolio and $\nabla_{t}$ is stock shares at time $t$. In a complete financial market, there are no risk-free arbitrage opportunities. Then

$$
\mathbb{E}\left(\mathrm{d} \Pi_{t}\right)=r \Pi_{t} \mathrm{~d} t
$$

No matter the jumps occur or not, by Merton assumptions $\ln \left(1+j_{t}\right) \sim \mathbb{N}\left[\ln \left(1+\mu_{j}\right)-(1 / 2) \sigma_{j}^{2}, \sigma_{j}^{2}\right]$ and model (17), the variance $\sigma_{j}^{2}$ exists surely. Either $P(X=x)>0$ for all $x>0$ or $P(X=x)=0$ for all $x>0$. In the first case, every positive point can be hit continuously in $X$ and this phenomenon is called creep [18]. In the second case, only jumps can occur (almost surely). In time interval $[t, t+\mathrm{d} t]$, the pricing satisfies the following hypothesis:

(i) If jumps can not occur, for the events $\omega_{1}$, by $V_{t}=$ $V\left(S_{t}, t\right)$ second-order differentiable, hence we can use Itô formula; then

$$
\begin{aligned}
& \mathrm{d} \Pi_{t}\left(\omega_{1}\right)=\mathrm{d} V_{t}-\nabla_{t} \mathrm{~d} S_{t}=\left[\frac{\partial V}{\partial t}\right. \\
& \left.+\left(H \sigma_{1}^{2} t^{2 H-1}+\frac{1}{2} \sigma_{2}^{2}+\frac{1}{2} \lambda \sigma_{3}^{2}\right) S^{2} \frac{\partial^{2} V}{\partial S^{2}}\right]_{\left(S_{t}, t\right)} \mathrm{d} t \\
& +\left(\frac{\partial V}{\partial S}-\nabla\right)_{\left(S_{t}, t\right)} \mathrm{d} S_{t} .
\end{aligned}
$$

(ii) If jumps can occur, for the events $\omega_{2}$, we have

$$
\begin{aligned}
\mathrm{d} \Pi_{t}\left(\omega_{2}\right) & =V\left(S_{t+}\right)-V\left(S_{t}, t\right)-\nabla_{t}\left(S_{t+}-S_{t}\right) \\
& =V\left[\left(1+j_{t}\right) S_{t}, t\right]-V\left(S_{t}, t\right)-\nabla_{t} j_{t} S_{t} .
\end{aligned}
$$

Then

$$
\begin{aligned}
& \mathbb{E}\left(\mathrm{d} \Pi_{t}\right)=r \Pi_{t} \mathrm{~d} t=(1-\lambda \mathrm{d} t)\left[\mathrm{d} \Pi_{t}\left(\omega_{1}\right)\right] \\
& +\lambda \mathrm{d} t\left[\mathrm{~d} \Pi_{t}\left(\omega_{2}\right)\right]=(1-\lambda \mathrm{d} t)\left\{\left[\frac{\partial V}{\partial t}\right.\right. \\
& \left.+\left(H \sigma_{1}^{2} t^{2 H-1}+\frac{1}{2} \sigma_{2}^{2}+\frac{1}{2} \lambda \sigma_{3}^{2}\right) S^{2} \frac{\partial^{2} V}{\partial S^{2}}\right]_{\left(S_{t}, t\right)} \mathrm{d} t \\
& \left.+\left(\frac{\partial V}{\partial S}-\nabla\right)_{\left(S_{t}, t\right)} \mathrm{d} S_{t}\right\}+\lambda \mathrm{d} t\left\{V\left[\left(1+j_{t}\right) S_{t}, t\right]\right. \\
& \left.\quad-V\left(S_{t}, t\right)-\nabla_{t} j_{t} S_{t}\right\} .
\end{aligned}
$$


Let $\nabla_{t}=\left.(\partial V / \partial S)\right|_{\left(S_{t}, t\right)}$ take expectations for $j_{t}$ on both sides of above equation, and cancel items of $\mathrm{d} t^{2}$; then we have a parabolic partial differential equation as follows:

$$
\begin{aligned}
\frac{\partial V}{\partial t}+ & \left(H \sigma_{1}^{2} t^{2 H-1}+\frac{1}{2} \sigma_{2}^{2}+\frac{1}{2} \lambda \sigma_{3}^{2}\right) S^{2} \frac{\partial^{2} V}{\partial S^{2}} \\
& +\left(r-\lambda \sigma_{3}\right) S \frac{\partial V}{\partial S} \\
& +\lambda \mathbb{E}\left[V\left(S\left(1+j_{t}\right), t\right)-V(S, t)\right]-(r+\lambda) V \\
= & 0,
\end{aligned}
$$

and then under the environment of mixed jump-diffusion fractional Brownian motion, lookback option pricing model can be expressed as a parabolic integral equation which contains expectation.

Hence we can use Taylor expansion and imitate proof of Lemma 8 , and by

$$
\left(\mathrm{d} P_{t}, \mathrm{~d} P_{t}\right)=\mathrm{d}\langle P, P\rangle(t)=\left(\mathrm{d} P_{t}\right)^{2}=\lambda \mathrm{d} t,
$$

and then by approximate that $\left(\mathrm{d} B_{t}^{H}\right)^{2}=2 H t^{2 H-1} \mathrm{~d} t,\left(\mathrm{~d} B_{t}\right)^{2}=$ $\mathrm{d} t,(\mathrm{~d} t)^{2}=0, \mathrm{~d} t \mathrm{~d} B_{t}=0$, we obtain

$$
\begin{aligned}
& \left(\mathrm{d} S_{t}\right)^{2}=\left[\mu S_{t} \mathrm{~d} t+\sigma_{1} S_{t} \mathrm{~d} B_{t}^{H}+\sigma_{2} S_{t} \mathrm{~d} B_{t}+\sigma_{3} S_{t} \mathrm{~d} P_{t}\right]^{2} \\
& =\mu^{2} S_{t}^{2}(\mathrm{~d} t)^{2}+\sigma_{1}^{2} S_{t}^{2}\left(\mathrm{~d} B_{t}^{H}\right)^{2}+\sigma_{2}^{2} S_{t}^{2}\left(\mathrm{~d} B_{t}\right)^{2} \\
& +\sigma_{3}^{2} S_{t}^{2}\left(\mathrm{~d} P_{t}\right)^{2}+2\left[\mu S_{t} \sigma_{1} S_{t} \mathrm{~d} B_{t}^{H} \mathrm{~d} t+\mu S_{t} \sigma_{2} S_{t} \mathrm{~d} B_{t} \mathrm{~d} t\right. \\
& +\mu S_{t} \sigma_{3} S_{t} \mathrm{~d} t \mathrm{~d} P_{t}+\sigma_{1} S_{t} \sigma_{2} S_{t} \mathrm{~d} B_{t}^{H} \mathrm{~d} B_{t} \\
& \left.+\sigma_{1} S_{t} \sigma_{3} S_{t} \mathrm{~d} B_{t}^{H} \mathrm{~d} P_{t}+\sigma_{2} S_{t} \sigma_{3} S_{t} \mathrm{~d} B_{t} \mathrm{~d} P_{t}\right] \\
& =2 H \sigma_{1}^{2} S_{t}^{2} t^{2 H-1} \mathrm{~d} t+\sigma_{2}^{2} S_{t}^{2} \mathrm{~d} t+\lambda \sigma_{3}^{2} S_{t}^{2} \mathrm{~d} t \\
& +o\left(\mathrm{~d} t \mathrm{~d} S_{t} \mathrm{~d} P_{t}\right) .
\end{aligned}
$$

Combine (25) with (26); then

$$
\begin{aligned}
\mathrm{d} V_{t} & =\frac{\partial V}{\partial t} \mathrm{~d} t+\frac{\partial V}{\partial S}\left[\mu S_{t} \mathrm{~d} t+\sigma_{1} S_{t} \mathrm{~d} B_{t}^{H}+\sigma_{2} S_{t} \mathrm{~d} B_{t}\right. \\
& \left.+\sigma_{3} S_{t} \mathrm{~d} P_{t}\right]+\frac{1}{2} \frac{\partial^{2} V}{\partial S^{2}} \cdot\left[2 H \sigma_{1}^{2} S_{t}^{2} t^{2 H-1} \mathrm{~d} t+\sigma_{2}^{2} S_{t}^{2} \mathrm{~d} t\right. \\
& \left.+\lambda \sigma_{3}^{2} S_{t}^{2} \mathrm{~d} t\right]=\left[\frac{\partial V}{\partial t}+\mu S_{t} \frac{\partial V}{\partial S}\right. \\
& \left.+\left(H \sigma_{1}^{2} S_{t}^{2} t^{2 H-1}+\frac{1}{2} \sigma_{2}^{2} S_{t}^{2}+\frac{1}{2} \lambda \sigma_{3}^{2} S_{t}^{2}\right) \frac{\partial^{2} V}{\partial S^{2}}\right] \mathrm{d} t \\
& +\sigma_{1} S_{t} \frac{\partial V}{\partial S} \mathrm{~d} B_{t}^{H}+\sigma_{2} S_{t} \frac{\partial V}{\partial S} \mathrm{~d} B_{t}+\sigma_{3} S_{t} \frac{\partial V}{\partial S} \mathrm{~d} P_{t} \\
& +\lambda \mathbb{E}\left[V\left(S\left(1+j_{t}\right), t\right)-V(S, t)\right] \mathrm{d} t .
\end{aligned}
$$

2.2. Wick-Itô-Skorohod Integral and Mixed Jump-Diffusion Fractional Stochastic Differential Equation.

Definition 10 (see [10]). Let $Y: \mathbb{R} \rightarrow(\delta)^{*}$ be a given function such that $Y_{t} \diamond B_{t}^{H}$ is $\mathrm{d} t$-integrable in $(\delta)^{*}$. The Wick-ItôSkorohod integral of $Y_{t}$ with respect to $B_{t}^{H}$ is given by

$$
\int_{\mathbb{R}} Y_{t} \mathrm{~d} B_{t}^{H}:=\int_{\mathbb{R}} Y_{t} \diamond B_{t}^{H} \mathrm{~d} t,
$$

where $\diamond$ is the Wick product and $B_{t}^{H}$ is the fractional Brownian motion.

Lemma 11 (fractional Girsanov formula I, see [10]). Let $\psi \epsilon$ $L^{p}\left(\mathbb{P}^{H}\right)$, for some $p>1$ and let $\gamma \in L_{\phi}^{2}(\mathbb{R}) \cap C(\mathbb{R}) \subset \delta^{\prime}(\mathbb{R})$. Let $\widetilde{\gamma}$ be defined by $\widetilde{\gamma}=\int_{\mathbb{R}} \phi(t, s) \mathrm{d}$ s. Then the map $\omega \rightarrow \psi(\omega+\widetilde{\gamma})$ belongs to $L^{\rho}\left(\mathbb{P}^{H}\right)$, for all $\rho<p$ and

$$
\begin{aligned}
& \int_{\delta^{\prime}(\mathbb{R})} \psi(\omega+\tilde{\gamma}) d \mathbb{P}^{H}(\omega) \\
& \quad=\int_{\delta^{\prime}(\mathbb{R})} \psi(\omega) \cdot \exp ^{\diamond}(\langle\omega, \gamma\rangle) \mathrm{dP}^{H}(\omega),
\end{aligned}
$$

where equip $\delta(\mathbb{R})$ with the inner product

$$
\langle\omega, \gamma\rangle_{H}:=\int_{\mathbb{R}} \int_{\mathbb{R}} \omega(s) \gamma(t) \psi(s, t) \mathrm{d} s \mathrm{~d} t,
$$

$$
\omega, \gamma \in \delta(\mathbb{R}) \text {. }
$$

Lemma $11^{*}$ (fractional Girsanov formula II, see [10]). Let $T>$ 0 , and let $\gamma$ be a continuous function with supp $\gamma \subset[0, T]$. Let $K$ be a function with supp $K \subset[0, T]$ and such that

$$
\langle K, f\rangle_{H}=\langle\gamma, f\rangle_{L^{2}(\mathbb{R})},
$$

$$
\forall f \in \delta(\mathbb{R}), \text { supp } f \subset[0, T],
$$

that is,

$$
\int_{\mathbb{R}} K(s) \phi(s, t) \mathrm{d} s=\gamma(t), \quad 0 \leq t \leq T .
$$

On the $\sigma$-algebra $\mathfrak{F}_{T}^{H}$ generated by $\left\{B_{s}^{H}: 0 \leq t \leq T\right\}$, define a probability measure $\mathbb{P}^{H, \gamma}$ by

$$
\frac{\mathrm{d} \mathbb{P}^{H, \gamma}}{\mathrm{d}^{\mathrm{P}}}=\exp ^{\diamond}(-\langle\omega, K\rangle),
$$

and then $\widehat{B}^{H}(t)=B_{t}^{H}+\int_{0}^{t} \gamma_{s} d s, 0 \leq t \leq T$, is a fBm under $\mathbb{P}^{H, \gamma}$.

Lemma 12 (Wick products on different white noise spaces, see [10]). Let $P=\mathbb{P}^{H}, Q=\mathbb{P}^{H, \gamma}$, and $\widehat{B}^{H}(t)=B_{t}^{H}+\int_{0}^{t} \gamma_{s} \mathrm{~d} s$. Let the Wick products corresponding to $P$ and $Q$ be denoted by $\diamond_{P}$ and $\diamond_{Q}$, respectively. Then

$$
F \diamond_{P} G=F \diamond_{Q} G
$$

for all $F, G \in(\delta)_{H}^{*}$. 
Suppose the price of the risky asset $S_{t}$ and interest rate $r_{t}$ satisfy the following equation:

$$
\begin{aligned}
\mathrm{d} S_{t} & =r_{t} S_{t} \mathrm{~d} t, \\
S_{0} & =1 .
\end{aligned}
$$

By Definition 10-Lemma 12 we can consider the mixed jump-diffusion fractional stochastic differential equation

$$
\begin{aligned}
& \mathrm{d} S_{t} \\
& =S_{t} \\
& \diamond\left[\left(r-q-\lambda \sigma_{3}\right) \mathrm{d} t+\sigma_{1} \mathrm{~d} B_{t}^{H}+\sigma_{2} \mathrm{~d} B_{t}+\sigma_{3} \mathrm{~d} P_{t}\right], \\
& S_{t}=S \text {, }
\end{aligned}
$$

where $r$ is the instantaneous expected return, $q \geq 0$ is the continuous dividend rate, $\sigma_{1}$ and $\sigma_{2}$ are denoted as in Lemma 5, and $\sigma_{3}$ is the unconditional expectation of $j_{t}$. Assume that $B_{t}, B_{t}^{H}$, and $P_{t}$ are independent.

Let $J$ be the path-dependent variable. For looking back put option on the maturity $T$, the stock price $S_{t}$ satisfies $S_{t} \leq$ $J_{t}=\max _{0 \leq t \leq T} S_{t}$; for looking back call option on the maturity $T$, the stock price $S_{t}$ satisfies $S_{t} \geq J_{t}=\min _{0 \leq t \leq T} S_{t}$. Thus, the looking back put option value $V\left(S_{t}, J_{t}, t\right)$ is the function of $S$, $J$, and $t$. Then we construct a riskless portfolio $\Pi=V-\nabla \diamond S$, select the appropriate variable $\nabla$, and make the investment portfolio $\Pi$ in the interval $(t, t+\mathrm{d} t)$ risk-free. Then $\mathrm{d} \Pi=$ $r(V-\nabla \diamond S) \mathrm{d} t$; according to Itô's formula we have

$$
\begin{gathered}
\mathrm{d} \Pi=\mathrm{d} V-\nabla \diamond \mathrm{d} S-\nabla q \diamond S \mathrm{~d} t=\frac{\partial V}{\partial t} \mathrm{~d} t+\frac{\partial V}{\partial J} \mathrm{~d} J+\frac{1}{2} \\
\cdot \lambda \sigma_{3}^{2} S^{2} \frac{\partial^{2} V}{\partial S^{2}} \mathrm{~d} t+\frac{\partial V}{\partial S} \mathrm{~d} S+H \sigma_{1}^{2} S^{2} t^{2 H-1} \frac{\partial^{2} V}{\partial S^{2}} \mathrm{~d} t+\frac{1}{2} \\
\cdot \sigma_{2}^{2} S^{2} \frac{\partial^{2} V}{\partial S^{2}} \mathrm{~d} t+\sigma_{3} \frac{\partial V}{\partial S} \mathrm{~d} P_{t}-\nabla \diamond \mathrm{d} S-\nabla q \diamond S \mathrm{~d} t \\
=\left[\frac{\partial V}{\partial t}+\left(H \sigma_{1}^{2} t^{2 H-1}+\frac{1}{2} \sigma_{2}^{2}+\frac{1}{2} \lambda \sigma_{3}^{2}\right) S^{2} \frac{\partial^{2} V}{\partial S^{2}}\right. \\
-\nabla q \diamond S] \mathrm{d} t+\frac{\partial V}{\partial J} \mathrm{~d} J+\sigma_{3} \frac{\partial V}{\partial S} \mathrm{~d} P_{t}+\left(\frac{\partial V}{\partial S}-\nabla\right)
\end{gathered}
$$

$\diamond \mathrm{d} S$.

Notice that $J_{t}$ is nondifferentiable about $t$, and define $J_{n}(t)=$ $\left[(1 / t) \int_{0}^{t}\left(S_{\tau}\right)^{n} \mathrm{~d} \tau\right]^{1 / n}$, such that approximate amount $J_{n}(t)$ is differentiable about $t$ and satisfies

$$
n J_{n}^{n-1}(t) \frac{\mathrm{d} J_{n}}{\mathrm{~d} t}=\frac{S_{t}^{n}-J_{n}^{n}(t)}{t} .
$$

Hence $S_{t}$ is continuous function with $t$, for $n \rightarrow \infty$, then $\lim _{n \rightarrow \infty} J_{n}(t)=\max _{0 \leq t \leq T} S_{t}=J_{t}$, and we get

$$
\begin{aligned}
\mathrm{d} \Pi & =\left[\frac{\partial V}{\partial t}+\left(H \sigma_{1}^{2} t^{2 H-1}+\frac{1}{2} \sigma_{2}^{2}+\frac{1}{2} \lambda \sigma_{3}^{2}\right) S^{2} \frac{\partial^{2} V}{\partial S^{2}}\right. \\
& -\nabla q \diamond S] \mathrm{d} t+\frac{\partial V}{\partial J} \mathrm{~d} J+\sigma_{3} \frac{\partial V}{\partial S} \mathrm{~d} P_{t}+\left(\frac{\partial V}{\partial S}-\nabla\right) \\
\diamond \mathrm{d} S=\left[\frac{\partial V}{\partial t}\right. & +\left(H \sigma_{1}^{2} t^{2 H-1}+\frac{1}{2} \sigma_{2}^{2}+\frac{1}{2} \lambda \sigma_{3}^{2}\right) S^{2} \frac{\partial^{2} V}{\partial S^{2}}+\frac{\partial V}{\partial J_{n}} \frac{d J_{n}}{d t} \\
& -\nabla q \diamond S] \mathrm{d} t+\sigma_{3} \frac{\partial V}{\partial S} \mathrm{~d} P_{t}+\left(\frac{\partial V}{\partial S}-\nabla\right) \diamond \mathrm{d} S .
\end{aligned}
$$

By $\nabla=\partial V / \partial S$, then

$$
\begin{aligned}
\frac{\partial V}{\partial t}+ & \left(H \sigma_{1}^{2} t^{2 H-1}+\frac{1}{2} \sigma_{2}^{2}+\frac{1}{2} \lambda \sigma_{3}^{2}\right) S^{2} \frac{\partial^{2} V}{\partial S^{2}} \\
& +\frac{\left(S / J_{n}\right)^{n-1} S-J_{n}}{n t} \frac{\partial V}{\partial J_{n}}+\left(r-q-\lambda \sigma_{3}\right) S \frac{\partial V}{\partial S} \\
& +\lambda \mathbb{E}\left[V\left(S\left(1+j_{t}\right), t\right)-V(S, t)\right]-(r+\lambda) V \\
= & 0,
\end{aligned}
$$

where $S \leq J_{n}$ and $\mathbb{E}$ is the expectation operator of $V$.

For fixed $(J, t)$, when $n \rightarrow \infty$, then $\left(\left(S / J_{n}\right)^{n-1} S-J_{n}\right) / n t \rightarrow$ 0 , since we obtain the general equations of lookback option under the environment of mixed jump-diffusion fraction Brownian motion as follows:

$$
\begin{aligned}
& \frac{\partial V}{\partial t}+\left(H \sigma_{1}^{2} t^{2 H-1}+\frac{1}{2} \sigma_{2}^{2}+\frac{1}{2} \lambda \sigma_{3}^{2}\right) S^{2} \frac{\partial^{2} V}{\partial S^{2}} \\
& \quad+\left(r-q-\lambda \sigma_{3}\right) S \frac{\partial V}{\partial S} \\
& \quad+\lambda \mathbb{E}\left[V\left(S\left(1+j_{t}\right), t\right)-V(S, t)\right]-(r+\lambda) V=0, \\
& V(S, J, t)=J-S,
\end{aligned}
$$

where $0 \leq S \leq J<\infty, 0 \leq t \leq T$, and the terminal condition is given by

$$
\left.\frac{\partial V}{\partial J}\right|_{S=J}=0
$$

\section{Main Results}

In this section we present the American lookback options factorization formula and the optimal exercise boundary. 
3.1. Fundamental Solution Derivation of the General Equations. Define $\mathfrak{G}(S, t ; \xi, T)$ as the fundamental solution of the following solution problem:

$$
\begin{aligned}
& \frac{\partial V}{\partial t}+\left(H \sigma_{1}^{2} t^{2 H-1}+\frac{1}{2} \sigma_{2}^{2}+\frac{1}{2} \lambda \sigma_{3}^{2}\right) S^{2} \frac{\partial^{2} V}{\partial S^{2}} \\
& \quad+\left(r-q-\lambda \sigma_{3}\right) S \frac{\partial V}{\partial S} \\
& \quad+\lambda \mathbb{E}\left[V\left(S\left(1+j_{t}\right), t\right)-V(S, t)\right]-(r+\lambda) V=0, \\
& V(S, T)=\delta(S-\xi),
\end{aligned}
$$

where $0<S<\infty, 0<\xi<\infty, 0<t<T$, and $\delta(x)$ is Dirac function.

Lemma 13 (see [29], Theorem 4.3). The price of a derivative on the stock price with a bounded payoff $f\left(S_{t}\right)$ is given by $D\left(t, S_{t}\right)$, where $D(t, S)$ is the solution of the PDE:

$$
\begin{aligned}
\frac{\partial D}{\partial t}+H \sigma^{2} S^{2} t^{2 H-1} \frac{\partial^{2} D}{\partial S^{2}}+r S \frac{\partial D}{\partial S}-r D & =0 \\
D(S, T) & =f(S) .
\end{aligned}
$$

Lemma 14. The solution of stochastic partial differential equations (43) and (44) is

$$
\mathfrak{G}(S, t ; \xi, T)=\sum_{n=0}^{\infty}\left\{\frac{\lambda^{n}(T-t)^{n} \exp [-\lambda(T-t)]}{n !} \mathscr{E}_{n}\right\}
$$

$$
\begin{aligned}
& \cdot \frac{\exp [-r(T-t)]}{\xi \sqrt{2 \pi\left[2 \sigma_{1}^{2}\left(T^{2 H}-t^{2 H}\right)+\sigma_{2}^{2}(T-t)+\lambda \sigma_{3}^{2}(T-t)\right]}} \\
& \cdot \exp \left\{-\frac{1}{2 \sigma_{1}^{2}\left(T^{2 H}-t^{2 H}\right)+\sigma_{2}^{2}(T-t)+\lambda \sigma_{3}^{2}(T-t)}\right. \\
& \cdot\left[\ln \frac{S \prod_{i=1}^{n}\left(1+j_{t_{i}}\right)}{\xi}+\left(r-q-\lambda \sigma_{3}\right)(T-t)\right. \\
& \left.\left.-\sigma_{1}^{2}\left(T^{2 H}-t^{2 H}\right)-\frac{1}{2}\left(\sigma_{2}^{2}+\lambda \sigma_{3}^{2}\right)(T-t)\right]^{2}\right\},
\end{aligned}
$$

where $\mathscr{C}_{n}$ denotes the expectation operator over the distribution of $\prod_{i=1}^{n}\left(1+j_{t_{i}}\right)$.

Proof. Let $x=\ln \left(\left(S \prod_{i=1}^{n}\left(1+j_{t_{i}}\right)\right) / \xi\right) \in \mathbb{R}, \mathfrak{W}=V \cdot \exp \{-r(T-$ $t)\}$; then solution problem of (43) and (44) is equivalent to the following solution problem:

$$
\begin{aligned}
& \frac{\partial \mathfrak{W}}{\partial t}-\left(H \sigma_{1}^{2} t^{2 H-1}+\frac{1}{2} \sigma_{2}^{2}+\frac{1}{2} \lambda \sigma_{3}^{2}\right) \frac{\partial^{2} \mathfrak{W}}{\partial x^{2}} \\
& -\left[\left(r-q-\lambda \sigma_{3}\right)-\left(H \sigma_{1}^{2} t^{2 H-1}+\frac{1}{2} \sigma_{2}^{2}+\frac{1}{2} \lambda \sigma_{3}^{2}\right)\right] \\
& \quad \frac{\partial \mathfrak{W}}{\partial x}-(r+\lambda) \mathfrak{W}=0 . \\
& \mathfrak{W}(x, T)=\frac{\exp \{-r(T-t)\}}{\xi} \delta(x),
\end{aligned}
$$

Let $\mathfrak{W}=\mathfrak{V} \cdot \exp \{\alpha(T, t)+\beta(T, t) x\}$, where

$$
\begin{aligned}
& \alpha(T, t)=-r(T-t)-\frac{\left[\left(r-q-\lambda \sigma_{3}\right)(T-t)-\sigma_{1}^{2}\left(T^{2 H}-t^{2 H}\right)-(1 / 2)\left(\sigma_{2}^{2}+\lambda \sigma_{3}^{2}\right)(T-t)\right]^{2}}{2 \sigma_{1}^{2}\left(T^{2 H}-t^{2 H}\right)+\sigma_{2}^{2}(T-t)+\lambda \sigma_{3}^{2}(T-t)}, \\
& \beta(T, t)=\frac{\sum_{n=0}^{\infty} P\left\{j_{t}=n\right\} \mathscr{E}_{n}}{\xi \sqrt{2 \pi\left[2 \sigma_{1}^{2}\left(T^{2 H}-t^{2 H}\right)+\sigma_{2}^{2}(T-t)+\lambda \sigma_{3}^{2}(T-t)\right]}}=\frac{\sum_{n=0}^{\infty}\left\{\lambda^{n}(T-t)^{n} \exp [-\lambda(T-t)](n !)^{-1} \mathscr{E}_{n}\right\}}{\xi \sqrt{2 \pi\left[2 \sigma_{1}^{2}\left(T^{2 H}-t^{2 H}\right)+\sigma_{2}^{2}(T-t)+\lambda \sigma_{3}^{2}(T-t)\right]}},
\end{aligned}
$$

here Poisson distribution with intensity $\lambda(T-t)$. Then by (47) we get

$$
\begin{aligned}
\frac{\partial \mathfrak{V}}{\partial t}-\left(H \sigma_{1}^{2} t^{2 H-1}+\frac{1}{2} \sigma_{2}^{2}+\frac{1}{2} \lambda \sigma_{3}^{2}\right) \frac{\partial^{2} \mathfrak{V}}{\partial x^{2}} \\
-\left[\left(r-q-\lambda \sigma_{3}\right)\right. \\
\left.-\left(H \sigma_{1}^{2} t^{2 H-1}+\frac{1}{2} \sigma_{2}^{2}+\frac{1}{2} \lambda \sigma_{3}^{2}\right)\right] \frac{\partial \mathfrak{V}}{\partial x}-(r+\lambda) \\
\cdot \mathfrak{V}=0,
\end{aligned}
$$

$$
\mathfrak{V}(x, 0)=\frac{\exp (-\beta x)}{\xi} \delta(x)=\frac{1}{\xi} \delta(x) .
$$

The solution of stochastic parabolic partial differential equations (49) and (50) can be written as

$$
\begin{aligned}
\mathfrak{V} & (x, \xi ; t, T) \\
& =\frac{1}{\left.\xi \sqrt{2 \pi\left[2 \sigma_{1}^{2}\left(T^{2 H}-t^{2 H}\right)+\sigma_{2}^{2}(T-t)+\lambda \sigma_{3}^{2}(T-t)\right.}\right]} \\
& \cdot \exp \left\{-\frac{x^{2}}{2 \sigma_{1}^{2}\left(T^{2 H}-t^{2 H}\right)+\sigma_{2}^{2}(T-t)+\lambda \sigma_{3}^{2}(T-t)}\right\} .
\end{aligned}
$$


Combining (51) with (48) we have

$\mathfrak{W}(x, \xi ; t, T)$

$$
\begin{aligned}
& =\frac{\sum_{n=0}^{\infty}\left\{\lambda^{n}(T-t)^{n} \exp [-\lambda(T-t)](n !)^{-1} \mathscr{C}_{n}\right\}}{\xi \sqrt{2 \pi\left[2 \sigma_{1}^{2}\left(T^{2 H}-t^{2 H}\right)+\sigma_{2}^{2}(T-t)+\lambda \sigma_{3}^{2}(T-t)\right]}} \\
& \cdot \exp \{[-r(T-t)] \\
& -\frac{1}{2 \sigma_{1}^{2}\left(T^{2 H}-t^{2 H}\right)+\sigma_{2}^{2}(T-t)+\lambda \sigma_{3}^{2}(T-t)}[x \\
& +\left(r-q-\lambda \sigma_{3}\right) \cdot(T-t)-\sigma_{1}^{2}\left(T^{2 H}-t^{2 H}\right) \\
& \left.\left.-\frac{1}{2}\left(\sigma_{2}^{2}+\lambda \sigma_{3}^{2}\right)(T-t)\right]^{2}\right\} .
\end{aligned}
$$

Substitute original variables $(S, t)$ in $(52)$; we obtain

$$
\begin{aligned}
& \text { GS }(S, t ; \xi, T)=\sum_{n=0}^{\infty}\left\{\frac{\lambda^{n}(T-t)^{n} \exp [-\lambda(T-t)]}{n !} \mathscr{E}_{n}\right\} \\
& \cdot \frac{\exp [-r(T-t)]}{\xi \sqrt{2 \pi\left[2 \sigma_{1}^{2}\left(T^{2 H}-t^{2 H}\right)+\sigma_{2}^{2}(T-t)+\lambda \sigma_{3}^{2}(T-t)\right]}} \\
& \cdot \exp \left\{-\frac{1}{2 \sigma_{1}^{2}\left(T^{2 H}-t^{2 H}\right)+\sigma_{2}^{2}(T-t)+\lambda \sigma_{3}^{2}(T-t)}\right. \\
& \cdot\left[\ln \frac{S \prod_{i=1}^{n}\left(1+j_{t_{i}}\right)}{\xi}+\left(r-q-\lambda \sigma_{3}\right)(T-t)\right. \\
& \left.\left.-\sigma_{1}^{2}\left(T^{2 H}-t^{2 H}\right)-\frac{1}{2}\left(\sigma_{2}^{2}+\lambda \sigma_{3}^{2}\right)(T-t)\right]^{2}\right\}
\end{aligned}
$$

Theorem 15. The fundamental solution $\mathfrak{G}(S, t ; \xi, T)$ is considered to be the function of $\xi$ and $\eta$, and $(\mathfrak{H}(S, t ; \xi, T)$ satisfies the adjoint equation of (43) and (44). If we note

$$
\mathfrak{G}(S, t ; \xi, \eta)=v(\xi, \eta),
$$

then $v(\xi, \eta)$ satisfies

$$
\begin{aligned}
& -\frac{\partial v}{\partial t}-\left(H \sigma_{1}^{2} t^{2 H-1}+\frac{1}{2} \sigma_{2}^{2}+\frac{1}{2} \lambda \sigma_{3}^{2}\right) S^{2} \frac{\partial^{2} v}{\partial S^{2}} \\
& -\left(r-q-\lambda \sigma_{3}\right) S \frac{\partial v}{\partial S} \\
& \quad-\lambda \mathbb{E}\left[v\left(S\left(1+j_{t}\right), t\right)-v(S, t)\right]-(r+\lambda) v=0, \\
& v(\xi, \eta)=\delta(\xi-S),
\end{aligned}
$$

where $0<S<\infty, 0<\xi<\infty, t<\eta$.
Corollary 16. By Lemmas 13 and 14 and Theorem 15, if one notes $\mathfrak{G H}^{*}(\xi, \eta ; S, t)$ is the fundamental solution of (55) and (56), then

$$
\mathfrak{G}(S, t ; \xi, \eta)=\mathfrak{G H}^{*}(\xi, \eta ; S, t)
$$

Proof of Theorem 15. For any $\epsilon>0$, consider integral as follows:

$$
\begin{aligned}
0= & \int_{0}^{\infty} \int_{t+\epsilon}^{\eta-\epsilon}\left[\mathfrak{G}^{*}(x, y ; S, t) \cdot \mathfrak{G}(x, y ; \xi, \eta)\right. \\
& -\left(\mathfrak{G}(x, y ; \xi, \eta) \cdot \mathfrak{G}^{*}(x, y ; S, t)\right] \mathrm{d} x \mathrm{~d} y \\
& =\int_{0}^{\infty} \mathrm{d} x \int_{t+\epsilon}^{\eta-\epsilon}\left\{\frac{\partial\left(\mathfrak{S}^{*} \mathfrak{G}\right)}{\partial y}+\left(H \sigma_{1}^{2} t^{2 H-1}+\frac{1}{2} \sigma_{2}^{2}\right.\right. \\
& \left.+\frac{1}{2} \lambda \sigma_{3}^{2}\right) \frac{\partial}{\partial x}\left(x^{2} \mathfrak{G}^{*} \frac{\partial \mathfrak{G}}{\partial x}\right) \\
& +\lambda \mathbb{E}\left[v\left(S\left(1+j_{t}\right), t\right)-v(S, t)\right]-\left(H \sigma_{1}^{2} t^{2 H-1}\right. \\
& \left.+\frac{1}{2} \sigma_{2}^{2}+\frac{1}{2} \lambda \sigma_{3}^{2}\right) \frac{\partial}{\partial x}\left[\mathfrak{G}_{\frac{\partial}{\partial x}}\left(x^{2} \mathfrak{G}^{*}\right)\right] \\
& +\left(r-q-\lambda \sigma_{3}\right) \frac{\partial}{\partial x}\left(x \mathfrak{G S}^{*}\right)-\lambda \mathbb{E}\left[v\left(S\left(1+j_{t}\right), t\right)\right. \\
& -v(S, t)]\} \mathrm{d} y .
\end{aligned}
$$

When $x \rightarrow 0$, we have

$$
\begin{aligned}
x^{2} \mathfrak{G}^{*} \frac{\partial \mathfrak{H}}{\partial x} & \longrightarrow 0, \\
\mathfrak{H} \frac{\partial}{\partial x}\left(x^{2} \mathfrak{H}^{*}\right) & \longrightarrow 0, \\
x \mathscr{H}^{*} \mathfrak{H}^{*} & \longrightarrow 0,
\end{aligned}
$$

since

$$
\begin{aligned}
& \int_{0}^{\infty} \mathfrak{G}^{*}(x, \eta-\epsilon ; S, t) \cdot \mathfrak{G}(x, \eta-\epsilon ; \xi, \eta) \mathrm{d} x \\
& \quad=\int_{0}^{\infty} \mathfrak{G}^{*}(x, t+\epsilon ; S, t) \cdot \mathfrak{G}(x, t+\epsilon ; \xi, \eta) \mathrm{d} x .
\end{aligned}
$$

Let $\epsilon \rightarrow 0$; by conditions (44) and (56) we get

$$
\begin{aligned}
& \int_{0}^{\infty} \mathfrak{S}^{*}(x, \eta ; S, t) \cdot \delta(x-\xi) \mathrm{d} x \\
& \quad=\int_{0}^{\infty} \delta(x-S) \cdot \mathfrak{H}(x, t ; \eta, \xi) \mathrm{d} x .
\end{aligned}
$$

Then

$$
\mathfrak{G}(S, t ; \xi, \eta)=\mathfrak{G}^{*}(\xi, \eta ; S, t)
$$


3.2. Early Exercise Boundary of American Lookback Option. Conze and Viswanathan [23] introduced a fractional or partial lookback option, where the strike is fixed at some fraction over (for a call) or below (for a put) the extreme value. Specifically, the payoffs for European lookback call and put with fractional floating strikes and maturity date $T$ are given, respectively, by $\left(S_{T}-a_{T}\right)^{+}$and $\left(b^{a} \mathbb{M}_{T}-S_{T}\right)^{+}$, where ๑ and $b$ are positive constants, allowing flexible adjustment of option premiums. To reduce option premiums, we assume that $₫ \geq 1$ and $0<\mathbb{b} \leq 1$. Given a finite time horizon $T>0$, let $\mathscr{C}=\mathscr{C}(t, S$, 田 $)$ be the value of the American fractional lookback call option at time $t \in[0, T]$. Note that the values of American and European call options are equal if the underlying asset pays no dividends. In the absence of arbitrage opportunities, the value $\mathscr{C}(t, S, \mathrm{~m})$ is a solution of an optimal stopping problem

$$
\begin{aligned}
\mathscr{C} & (t, S, \text { m) } \\
& =\sup _{T_{t} \in[t, T]} \mathbb{E}\left[\exp \left\{-r\left(T_{t}-t\right)\right\}\left(S_{T_{t}}-\operatorname{amc}_{T_{t}}\right)^{+} \mid S_{t}\right. \\
& \left.=S, \operatorname{man}_{t}=\operatorname{mo}\right],
\end{aligned}
$$

where $T_{t}$ is a stopping time of the filtration $\mathbb{F}$ and the conditional expectation is calculated under the risk-neutral probability measure $\mathbb{P}$. The random variable $T_{t}^{*} \in[t, T]$ is called an optimal stopping time if it gives the supremum value of the right-hand side of (63). It is clear from (63) that $\mathscr{C}$ is nondecreasing in $S$ and nonincreasing in $t, 0$, and $₫$. Solving the optimal stopping problem (63) is equivalent to finding the points $\left(t, S_{t}, \mathrm{mu}_{t}\right)$ for which early exercise before maturity is optimal. Let

$$
\mathfrak{D}=\left\{(t, S, \mathbb{m}) \in[0, T] \times[m, \infty) \times \mathbb{R}_{+}\right\}
$$

be the whole domain, and let $\mathfrak{E}$ and $\mathfrak{C}$ denote the exercise region and continuation region, respectively. In terms of the value function $\mathscr{C}(t, S$, mo $)$, the exercise region $\mathfrak{E}$ is defined by

$$
\mathfrak{E}=\left\{(t, S, \text { m) }) \mid \mathscr{C}(t, S, \text { m })=(S-\underset{m}{ })^{+}\right\},
$$

for which the optimal stopping time $T_{t}^{*}$ satisfies

$$
T_{t}^{*}=\inf \left\{\eta \in[t, T] \mid\left(\eta, S_{\eta}, \mathrm{ma}_{\eta}\right) \in \mathfrak{E}\right\}
$$

The continuation region $\mathfrak{E}$ is the complement of $\mathcal{S}$ in $\mathfrak{D}$, such as

$$
\mathfrak{S}=\left\{(t, S, \text { m }) \mid \mathscr{C}(t, S, \text { m })>(S-\mathbb{a r m})^{+}\right\}
$$

The boundary that separates $\mathcal{S}$ from $\mathbb{E}$ is referred to as the early exercise boundary, which is defined by

$$
\bar{S}(t, m)=\sup \{S \geq m \mid(t, S, m) \in \mathbb{E}\}, \quad t \in[0, T] .
$$

At the early exercise boundary $[\bar{S}(t, m)]_{t \in[0, T]}$, the American fractional lookback call option would be optimally exercised. In terms of $S(t, 0)$, the continuation region $\boldsymbol{C}$ can be represented as

$$
\mathfrak{S}=\{(t, S, m) ; m \leq S<\bar{S}(t, m)\} .
$$

Let $V(S, J, t)$ be the lookback option price at time $t$ with stock price $S$ and path-dependent variable $J$. Using argument similar to Section 2.2, it can be shown that the American fractional lookback option price solves the following stochastic partial differential equations:

$$
\begin{aligned}
& 0=\frac{\partial V}{\partial t}+\left(H \sigma_{1}^{2} t^{2 H-1}+\frac{1}{2} \sigma_{2}^{2}+\frac{1}{2} \lambda \sigma_{3}^{2}\right) S^{2} \frac{\partial^{2} V}{\partial S^{2}}+\left(r-q-\lambda \sigma_{3}\right) S \frac{\partial V}{\partial S}-(r+\lambda) V \\
& + \begin{cases}\lambda \mathbb{E}\left[V\left(S\left(1+j_{t}\right), \min \left\{J, S\left(1+j_{t}\right)\right\}, t\right)\right], & \text { for lookback call option, } \\
\lambda \mathbb{E}\left[V\left(S\left(1+j_{t}\right), \max \left\{J, S\left(1+j_{t}\right)\right\}, t\right)\right], & \text { for lookback put option, }\end{cases} \\
& V(S, J, t)=J-S
\end{aligned}
$$

where $0 \leq t \leq T$, and satisfies an order continuous differentiable on domain

$$
\begin{aligned}
& \Sigma \\
& = \begin{cases}\{(S, J): 0<J \leq S<\infty\}, & \text { for lookback call option, } \\
\{(S, J): 0<S \leq J<\infty\}, & \text { for lookback put option, }\end{cases}
\end{aligned}
$$

and the terminal condition is given by

$$
\left.\frac{\partial V}{\partial J}\right|_{S=J}=0 .
$$

We define the differential operator $\mathscr{L}_{t, S}$ by

$$
\begin{gathered}
\mathscr{L}_{t, S}=\frac{\partial}{\partial t}+\left(H \sigma_{1}^{2} t^{2 H-1}+\frac{1}{2} \sigma_{2}^{2}+\frac{1}{2} \lambda \sigma_{3}^{2}\right) S^{2} \frac{\partial^{2}}{\partial S^{2}} \\
+\left(r-q-\lambda \sigma_{3}\right) S \frac{\partial}{\partial S}-(r+\lambda) .
\end{gathered}
$$

Then the free boundary problem can be written in a linear complementary form as

$$
\left[\mathscr{L}_{t, S} \mathscr{C}(t, S, m)\right] \cdot\left[\mathscr{C}(t, S, \text { m })-(S-\mathbb{a m})^{+}\right]=0,
$$




$$
\begin{array}{r}
\mathscr{L}_{t, S} \mathscr{C}(t, S, \text { m) }) \leq 0, \\
\mathscr{C}(t, S, \text { m })-(S-\text { am }))^{+} \geq 0
\end{array}
$$

together with auxiliary conditions

$$
\begin{aligned}
\mathscr{C}(T, S, m)-(S-\underset{m}{ }))^{+} & =0, \\
\lim _{S \downarrow m} \frac{\partial \mathscr{C}}{\partial m} & =0 .
\end{aligned}
$$

For the free boundary $[\bar{S}(t, m)]_{t \in[0, T]}$, this problem is equivalent to solving the Black-Scholes-Merton partial differential equations

$$
\mathscr{L}_{t, S} \mathscr{C}(t, S, \text { m) })=0, \quad \text { 必 } \leq S<\bar{S}(t, \text { m) }),
$$

together with the boundary conditions

$$
\begin{aligned}
& \lim _{S \uparrow \bar{S}} \mathscr{C}(t, S, m)=\bar{S}(t, m)-₫ a m, \\
& \lim _{S \uparrow \bar{S}} \frac{\partial \mathscr{C}}{\partial S}=1 \\
& \lim _{S \downarrow m} \frac{\partial \mathscr{C}}{\partial m m}=0 .
\end{aligned}
$$

and the terminal condition

$$
\mathscr{C}(T, S, \mathbb{m})=(S-\mathbb{a m})^{+} .
$$

In the same way as in the call case, by (63), (70), (71), (72), (76), (77), and (78), we can formulate the put case: Let $\mathscr{P}=$ $\mathscr{P}(t, S$, m $)$ be the value of the American fractional lookback call option at time $t \in[0, T]$. The value $\mathscr{P}(t, S, \mathbb{M})$ is a solution of an optimal stopping problem

$$
\begin{aligned}
& \mathscr{P}(t, S, \mathbb{M}) \\
& \quad=\sup _{T_{t} \in[t, T]} \mathbb{E}\left[\exp \left\{-r\left(T_{t}-t\right)\right\}\left(\mathfrak{b}_{\mathbb{M}_{T_{t}}}-S_{T_{t}}\right)^{+} \mid S_{t}\right. \\
& \left.\quad=S, \mathbb{M}_{t}=\mathbb{M}\right],
\end{aligned}
$$

and $\mathscr{P}(t, S, \mathbb{M})$ satisfies the same PDE as (76); then

$$
\mathscr{L}_{t, S} \mathscr{P}(t, S, \mathbb{M})=0, \quad \underline{S}(t, \mathbb{M})<S \leq \mathbb{M},
$$

where $[\underline{S}(t, \mathbb{M})]_{t \in[0, T]}$ is the early exercise boundary for put. The boundary conditions for put are

$$
\begin{aligned}
\lim _{S \downarrow \underline{S}} \mathscr{P}(t, S, \mathbb{M}) & =\mathfrak{b M}-\underline{S}(t, \mathbb{M}), \\
\lim _{S \downarrow \underline{S} \underline{\underline{S}}} \frac{\partial \mathscr{P}}{\partial S} & =-1, \\
\lim _{S \uparrow \mathbb{M}} \frac{\partial \mathscr{P}}{\partial \mathbb{M}} & =0 .
\end{aligned}
$$

and the terminal condition is given by

$$
\mathscr{P}(T, S, \mathbb{M})=(\mathbb{b} \mathbb{M}-S)^{+} .
$$

3.3. Optimal Exercise Boundary. It is well known that the value of an American fractional option can be represented as the sum of the value of the corresponding European option and the early exercise premium. For American fractional lookback options, Lai and Lim [28] proved that the value has such a decomposition and that the premium has an integral representation. Applying the same solution method as in Theorem 15 to the $\operatorname{PDE}(82)$ for $\mathscr{P}(t, S, \mathbb{M})$, we can obtain the early exercise representation $V(t, S, \mathbb{M})$ under our mixed jump-diffusion fractional Brownian motion environment, which is shown in the following theorem.

Theorem 17. Let $V(t, S, \mathbb{M})$ be American fractional lookback put option; then

$$
V(t, S, \mathbb{M})=V_{E}(t, S, \mathbb{M})+e(t, S, \mathbb{M}),
$$

where $V_{E}(t, S, \mathbb{M})$ is price of the hedging portfolio of the equivalent European option, and

$$
\begin{gathered}
V_{E}(t, S, \mathbb{M})=\sum_{n=0}^{\infty}\left\{\frac{\lambda^{n}(T-t)^{n} \exp [-\lambda(T-t)]}{n !} \mathscr{E}_{n}\right\} \\
\cdot\left[\mathbb{b} \mathbb{M} \exp \{-r(T-t)\} \mathbb{N}\left(-\widehat{d}_{2}\right)-S \prod_{i=1}^{n}\left(1+j_{t_{i}}\right)\right. \\
\left.\cdot \exp \left\{-\left(q+\lambda \sigma_{3}\right)(T-t)\right\} \mathbb{N}\left(-\widehat{d}_{1}\right)\right],
\end{gathered}
$$

where $\mathscr{E}_{n}$ denotes the expectation operator over the distribution of $\prod_{i=1}^{n}\left(1+j_{t_{i}}\right), \mathbb{M}$ is contractual strike price, $\mathbb{b}$ is positive constants, $\mathbb{N}(\cdot)$ is the cumulative normal distribution function, and

$$
\begin{aligned}
\widehat{d}_{1} & =\left[\ln \frac{S \prod_{i=1}^{n}\left(1+j_{t_{i}}\right)}{\xi}+\left(r-q-\lambda \sigma_{3}\right)(T-t)\right. \\
& \left.+\sigma_{1}^{2}\left(T^{2 H}-t^{2 H}\right)+\frac{1}{2}\left(\sigma_{2}^{2}+\lambda \sigma_{3}^{2}\right) \cdot(T-t)\right] \\
& \cdot\left(\sqrt{2 \sigma_{1}^{2}\left(T^{2 H}-t^{2 H}\right)+\sigma_{2}^{2}(T-t)+\lambda \sigma_{3}^{2}(T-t)}\right)^{-1}, \\
\widehat{d}_{2} & =\widehat{d}_{1} \\
& -\sqrt{2 \sigma_{1}^{2}\left(T^{2 H}-t^{2 H}\right)+\sigma_{2}^{2}(T-t)+\lambda \sigma_{3}^{2}(T-t)},
\end{aligned}
$$

$e(t, S, \mathbb{M})$ is early exercise premium, and its explicit integral can be represented by

$$
\begin{aligned}
& e(t, S, \mathbb{M}) \\
& \quad=\int_{t}^{T} \mathrm{~d} \eta \int_{0}^{S_{\eta}}\left[\mathbb{b M M} r-\left(q+\lambda \sigma_{3}\right) \xi\right] \cdot \mathfrak{G}(S, t ; \xi, \eta) \mathrm{d} \xi .
\end{aligned}
$$

Proof. Suppose $V(t, S, \mathbb{M})$ satisfies an order continuous differentiable on domain $\Sigma: \Sigma=\{0 \leq S \leq \mathbb{M}<\infty, 0 \leq t<T\}$, 
and $V(t, S, \mathbb{M})$ is piecewise continuous on domain $\Sigma$, and the second-order derivative exists; then

$$
\begin{aligned}
- & \mathbb{Q} V(t, S, \mathbb{M}) \\
& = \begin{cases}0, & (t, S, \mathbb{M}) \in \Sigma_{1}, \\
\mathbb{b} \mathbb{M} r-\left(q+\lambda \sigma_{3}\right) S, & (t, S, \mathbb{M}) \in \Sigma_{2},\end{cases}
\end{aligned}
$$

where $\mathfrak{Q}$ is the Black-Scholes operator.

Multiplying both sides of (87) to $\boldsymbol{G}^{*}(\xi, \eta ; S, t)$ and quadrature on domain $\{0 \leq \xi \leq \mathbb{M}<\infty, t+\epsilon \leq \eta \leq T\}$, for $\Sigma_{2}=\left\{0 \leq \xi \leq \mathbb{M} \leq S_{\eta}, 0 \leq \eta<T\right\}$ and $S_{\eta}$ is continuous monotonous of the asset price, then

$$
\begin{aligned}
& \int_{t+\epsilon}^{T} \mathrm{~d} \eta \int_{0}^{S_{\eta}}\left[\mathrm{b} \mathbb{M} r-\left(q+\lambda \sigma_{3}\right) \xi\right] \cdot \mathfrak{G}^{*}(\xi, \eta ; S, t) \mathrm{d} \xi \\
& =-\int_{t+\epsilon}^{T} \mathrm{~d} \eta \int_{0}^{\infty} \mathfrak{G}^{*}(\xi, \eta ; S, t) \mathfrak{Q} V \mathrm{~d} \xi=-\int_{t+\epsilon}^{T} \mathrm{~d} \eta \\
& \cdot \int_{0}^{\infty}\left[\mathfrak{G}^{*}(\xi, \eta ; S, t) \mathfrak{Q V}(\eta, \xi, \mathbb{M})\right. \\
& \left.-V(\eta, \xi, \mathbb{M}) \mathfrak{Q}^{*} \mathfrak{G}^{*}(\xi, \eta ; S, t)\right] \mathrm{d} \xi=-\int_{t+\epsilon}^{T} \mathrm{~d} \eta \\
& \cdot \int_{0}^{\infty}\left\{\frac{\partial\left(\mathfrak{G}^{*} V\right)}{\partial \eta}\right. \\
& +\left(H \sigma_{1}^{2} t^{2 H-1}+\frac{1}{2} \sigma_{2}^{2}+\frac{1}{2} \lambda \sigma_{3}^{2}\right) \frac{\partial}{\partial \xi}\left(\xi^{2} \mathfrak{G}^{*} \frac{\partial V}{\partial \xi}\right) \\
& +\lambda \mathbb{E}\left[V\left(t, S\left(1+j_{t}\right), \mathbb{M}\right)-V(t, S, \mathbb{M})\right] \\
& -\left(H \sigma_{1}^{2} t^{2 H-1}+\frac{1}{2} \sigma_{2}^{2}+\frac{1}{2} \lambda \sigma_{3}^{2}\right) \frac{\partial}{\partial \xi} \\
& \cdot\left[V \frac{\partial}{\partial \xi}\left(\xi^{2} \mathfrak{G}^{*}\right)\right]+\left(r-q-\lambda \sigma_{3}\right) \frac{\partial}{\partial \xi}\left(\xi V \mathfrak{G}^{*}\right) \\
& \left.-\lambda \mathbb{E}\left[V\left(t, S\left(1+j_{t}\right), \mathbb{M}\right)-V(t, S, \mathbb{M})\right]\right\} \mathrm{d} \xi,
\end{aligned}
$$

$$
\begin{aligned}
S_{t} & =\mathbb{b M M}+S \exp \left\{-\left(q+\lambda \sigma_{3}\right)(T-t)\right\} \cdot \mathbb{N}\left\{-\left[-\ln \frac{S \prod_{i=1}^{n}\left(1+j_{t_{i}}\right)}{\mathfrak{b M M}}+\left(r-q-\lambda \sigma_{3}\right)(T-t)+\sigma_{1}^{2}\left(T^{2 H}-t^{2 H}\right)+\frac{1}{2}\left(\sigma_{2}^{2}\right.\right.\right. \\
& \left.\left.\left.+\lambda \sigma_{3}^{2}\right)(T-t)\right] \cdot\left[\sqrt{2 \sigma_{1}^{2}\left(T^{2 H}-t^{2 H}\right)+\sigma_{2}^{2}(T-t)+\lambda \sigma_{3}^{2}(T-t)}\right]^{-1}\right\}-\mathbb{b M M} \exp \{-r(T-t)\} \\
& \cdot \sum_{n=0}^{\infty}\left\{\frac{\lambda^{n}(T-t)^{n} \exp [-\lambda(T-t)]}{n !} \mathscr{E}_{n}\right\} \cdot \mathbb{N}\left\{-\left[-\ln \frac{S \prod_{i=1}^{n}\left(1+j_{t_{i}}\right)}{\mathfrak{b M M}}+\left(r-q-\lambda \sigma_{3}\right)(T-t)-\sigma_{1}^{2}\left(T^{2 H}-t^{2 H}\right)\right.\right.
\end{aligned}
$$

where $\mathbf{Q}^{*}$ is the adjoint Black-Scholes operator, and when $\xi \rightarrow 0$, we have

$$
\begin{array}{r}
\xi^{2} \mathfrak{G}^{*} \frac{\partial V}{\partial \xi} \\
V \frac{\partial}{\partial \xi}\left(\xi^{2} \mathfrak{S}^{*}\right) \longrightarrow 0, \\
\xi V \mathscr{H}^{*} \longrightarrow 0,
\end{array}
$$

and hence

$$
\begin{aligned}
\int_{0}^{\infty} & \mathscr{G}^{*}(\xi, t+\epsilon ; S, t) V(t+\epsilon, \xi, \mathbb{M}) \mathrm{d} \xi \\
\quad= & \int_{0}^{\infty} \mathfrak{G}^{*}(\xi, T ; S, t) V(T, \xi, \mathbb{M}) \mathrm{d} \xi+\int_{t+\epsilon}^{T} \mathrm{~d} \eta \\
& \cdot \int_{0}^{S_{\eta}}\left[\mathbb{b} \mathbb{M} r-\left(q+\lambda \sigma_{3}\right) \xi\right] \mathfrak{G}^{*}(\xi, \eta ; S, t) \mathrm{d} \xi .
\end{aligned}
$$

Let $\epsilon \rightarrow 0$; by (56) and Corollary 16, we obtain

$$
\begin{aligned}
V(t, S, \mathbb{M}) \\
=\int_{0}^{\infty} \mathfrak{G}(S, t ; \xi, T)(\mathbb{b} \mathbb{M}-\xi)^{+} \mathrm{d} \xi \\
\quad+\int_{t}^{T} \mathrm{~d} \eta \int_{0}^{S_{\eta}}\left[\mathbb{b} \mathbb{M} r-\left(q+\lambda \sigma_{3}\right) \xi\right] \mathfrak{G}(S, t ; \xi, \eta) \mathrm{d} \xi \\
=V_{E}(t, S, \mathbb{M})+e(t, S, \mathbb{M}) .
\end{aligned}
$$

Remark 18. If the optimal exercise boundary is given by $S=$ $S_{t}$, then pricing of American fractional lookback options can be represented as (83)-(86).

Remark 19. This results show that American fractional lookback options are equal to the option of a hedging portfolio: a risk premium associated with the European values plus the early exercise premium. Using the similar techniques, the optimal exercise boundary can be given by the following corollary.

Corollary 20. The optimal exercise boundary of looking back American fractional put options is defined by $S=S_{t}, 0 \leq t<T$, and then $S=S_{t}$ satisfy the following Volterra integral equation 


$$
\begin{aligned}
& \left.\left.-\frac{1}{2}\left(\sigma_{2}^{2}+\lambda \sigma_{3}^{2}\right)(T-t)\right] \cdot\left[\sqrt{2 \sigma_{1}^{2}\left(T^{2 H}-t^{2 H}\right)+\sigma_{2}^{2}(T-t)+\lambda \sigma_{3}^{2}(T-t)}\right]^{-1}\right\}-\operatorname{baM} r \int_{t}^{T} \exp \{-r(\eta-t)\} \\
& \cdot \sum_{n=0}^{\infty}\left\{\frac{\lambda^{n}(\eta-t)^{n} \exp [-\lambda(\eta-t)]}{n !} \mathscr{E}_{n}\right\} \cdot\{1 \\
& -\mathbb{N}\left[\left(\ln \frac{S \prod_{i=1}^{n}\left(1+j_{t_{i}}\right)}{S_{\eta}}+\left(r-q-\lambda \sigma_{3}\right)(\eta-t)-\sigma_{1}^{2}\left(\eta^{2 H}-t^{2 H}\right)-\frac{1}{2}\left(\sigma_{2}^{2}+\lambda \sigma_{3}^{2}\right)(\eta-t)\right)\right. \\
& \left.\left.\cdot\left(\sqrt{2 \sigma_{1}^{2}\left(\eta^{2 H}-t^{2 H}\right)+\sigma_{2}^{2}(\eta-t)+\lambda \sigma_{3}^{2}(\eta-t)}\right)^{-1}\right]\right\} \mathrm{d} \eta+\left(q+\lambda \sigma_{3}\right) S \int_{t}^{T} \exp \left\{-\left(q+\lambda \sigma_{3}\right)(\eta-t)\right\} \\
& \cdot \sum_{n=0}^{\infty}\left\{\frac{\lambda^{n}(\eta-t)^{n} \exp [-\lambda(\eta-t)]}{n !} \mathscr{E}_{n}\right\} \cdot\{1 \\
& -\mathbb{N}\left[\left(\ln \frac{S \prod_{i=1}^{n}\left(1+j_{t_{i}}\right)}{S_{\eta}}+\left(r-q-\lambda \sigma_{3}\right)(\eta-t)+\sigma_{1}^{2}\left(\eta^{2 H}-t^{2 H}\right)+\frac{1}{2}\left(\sigma_{2}^{2}+\lambda \sigma_{3}^{2}\right)(\eta-t)\right)\right. \\
& \left.\left.\cdot\left(\sqrt{2 \sigma_{1}^{2}\left(\eta^{2 H}-t^{2 H}\right)+\sigma_{2}^{2}(\eta-t)+\lambda \sigma_{3}^{2}(\eta-t)}\right)^{-1}\right]\right\} \mathrm{d} \eta,
\end{aligned}
$$

where $\mathbb{N}(\cdot)$ is the cumulative normal distribution function and $S_{\eta} \geq \mathbb{M}>0,0<\eta \leq T$.

Proof. By (86) and (50), $e(S, t)$ can be represented by

$$
\begin{aligned}
e(t, S, \mathbb{M})=\int_{t}^{T} \mathrm{~d} \eta \int_{0}^{S_{\eta}}\left[\mathrm{b} \mathbb{M} r-\left(q+\lambda \sigma_{3}\right) \xi\right] & \cdot \sum_{n=0}^{\infty}\left\{\frac{\lambda^{n}(T-t)^{n} \exp [-\lambda(T-t)]}{n !} \mathscr{E}_{n}\right\} \\
\cdot & \frac{\exp \{-r(T-t)\}}{\xi \sqrt{2 \pi\left[2 \sigma_{1}^{2}\left(T^{2 H}-t^{2 H}\right)+\sigma_{2}^{2}(T-t)+\lambda \sigma_{3}^{2}(T-t)\right]}} \\
\cdot & \exp \left\{-\frac{1}{2 \sigma_{1}^{2}\left(T^{2 H}-t^{2 H}\right)+\sigma_{2}^{2}(T-t)+\lambda \sigma_{3}^{2}(T-t)}\right. \\
& \cdot\left[\ln \frac{S \prod_{i=1}^{n}\left(1+j_{t_{i}}\right)}{\xi}+\left(r-q-\lambda \sigma_{3}\right)(T-t)\right. \\
& \left.\left.-\sigma_{1}^{2}\left(T^{2 H}-t^{2 H}\right)-\frac{1}{2}\left(\sigma_{2}^{2}+\lambda \sigma_{3}^{2}\right)(T-t)\right]^{2}\right\} \mathrm{d} \xi .
\end{aligned}
$$

$$
\begin{aligned}
x^{*} & =\frac{1}{\sqrt{2 \sigma_{1}^{2}\left(\eta^{2 H}-t^{2 H}\right)+\sigma_{2}^{2}(\eta-t)+\lambda \sigma_{3}^{2}(\eta-t)}} \\
& \cdot\left[\ln \frac{S \prod_{i=1}^{n}\left(1+j_{t_{i}}\right)}{\xi}+\left(r-q-\lambda \sigma_{3}\right)(\eta-t)\right. \\
& \left.-\sigma_{1}^{2}\left(\eta^{2 H}-t^{2 H}\right)-\frac{1}{2}\left(\sigma_{2}^{2}+\lambda \sigma_{3}^{2}\right)(\eta-t)\right],
\end{aligned}
$$

$\mathrm{d} x^{*}$

$$
=-\frac{1}{\xi \sqrt{2 \sigma_{1}^{2}\left(T^{2 H}-t^{2 H}\right)+\sigma_{2}^{2}(T-t)+\lambda \sigma_{3}^{2}(T-t)}} \mathrm{d} \xi,
$$

and then

$$
\begin{aligned}
& e(t, S, \mathbb{M})=\frac{b \mathbb{M} r}{\sqrt{2 \pi}} \int_{t}^{T} \exp \{-r(\eta-t)\} \sum_{n=0}^{\infty}\left\{\frac{\lambda^{n}(\eta-t)^{n} \exp [-\lambda(\eta-t)]}{n !} \mathscr{E}_{n}\right\} \mathrm{d} \eta \\
& \cdot \int_{\left(\ln \left(\left(S \prod_{i=1}^{n}\left(1+j_{t_{i}}\right)\right) / S_{\eta}\right)+\left(r-q-\lambda \sigma_{3}\right)(\eta-t)-\sigma_{1}^{2}\left(\eta^{2 H}-t^{2 H}\right)-(1 / 2)\left(\sigma_{2}^{2}+\lambda \sigma_{3}^{2}\right)(\eta-t)\right) / \sqrt{2 \sigma_{1}^{2}\left(\eta^{2 H}-t^{2 H}\right)+\sigma_{2}^{2}(\eta-t)+\lambda \sigma_{3}^{2}(\eta-t)}}^{\infty} \exp \left\{-x^{2}\right\} \mathrm{d} x \\
& \quad-\frac{\left(q+\lambda \sigma_{3}\right) S}{\sqrt{2 \pi}} \int_{t}^{T} \exp \left\{-\left(q+\lambda \sigma_{3}\right)(\eta-t)\right\} \cdot \sum_{n=0}^{\infty}\left\{\frac{\lambda^{n}(\eta-t)^{n} \exp [-\lambda(\eta-t)]}{n !} \mathscr{E}_{n}\right\} \mathrm{d} \eta
\end{aligned}
$$




$$
\begin{aligned}
& \cdot \int_{\left(\ln \left(\left(S \prod_{i=1}^{n}\left(1+j_{t_{i}}\right)\right) / S_{\eta}\right)+\left(r-q-\lambda \sigma_{3}\right)(\eta-t)-\sigma_{1}^{2}\left(\eta^{2 H}-t^{2 H}\right)-(1 / 2)\left(\sigma_{2}^{2}+\lambda \sigma_{3}^{2}\right)(\eta-t)\right) / \sqrt{2 \sigma_{1}^{2}\left(\eta^{2 H}-t^{2 H}\right)+\sigma_{2}^{2}(\eta-t)+\lambda \sigma_{3}^{2}(\eta-t)}}^{\infty} \exp \left\{-\frac{x^{2}}{2}\right. \\
& \left.+\left[\sqrt{2 \sigma_{1}^{2}\left(\eta^{2 H}-t^{2 H}\right)+\sigma_{2}^{2}(\eta-t)+\lambda \sigma_{3}^{2}(\eta-t)}\right] x\right\} \mathrm{d} x=\mathbb{b} \mathbb{M} r \int_{t}^{T} \exp \{-r(\eta-t)\} \\
& \cdot \sum_{n=0}^{\infty}\left\{\frac{\lambda^{n}(\eta-t)^{n} \exp [-\lambda(\eta-t)]}{n !} \mathscr{E}_{n}\right\} \cdot\{1 \\
& \left.-\mathbb{N}\left[\frac{\ln \left(\left(S \prod_{i=1}^{n}\left(1+j_{t_{i}}\right)\right) / S_{\eta}\right)+\left(r-q-\lambda \sigma_{3}\right)(\eta-t)-\sigma_{1}^{2}\left(\eta^{2 H}-t^{2 H}\right)-\left(\sigma_{2}^{2}+\lambda \sigma_{3}^{2}\right)(\eta-t) / 2}{2 \sigma_{1}^{2}\left(\eta^{2 H}-t^{2 H}\right)+\sigma_{2}^{2}(\eta-t)+\lambda \sigma_{3}^{2}(\eta-t)}\right]\right\} \mathrm{d} \eta-(q \\
& \left.+\lambda \sigma_{3}\right) S \int_{t}^{T} \exp \left\{-\left(q+\lambda \sigma_{3}\right)(\eta-t)\right\} \sum_{n=0}^{\infty}\left\{\frac{\lambda^{n}(\eta-t)^{n} \exp [-\lambda(\eta-t)]}{n !} \mathscr{E}_{n}\right\} \mathrm{d} \eta \\
& \cdot \int_{\left(\ln \left(\left(S \prod_{i=1}^{n}\left(1+j_{t_{i}}\right)\right) / S_{\eta}\right)+\left(r-q-\lambda \sigma_{3}\right)(\eta-t)-\sigma_{1}^{2}\left(\eta^{2 H}-t^{2 H}\right)-(1 / 2)\left(\sigma_{2}^{2}+\lambda \sigma_{j}^{2}\right)(\eta-t)\right) / \sqrt{2 \sigma_{1}^{2}\left(\eta^{2 H}-t^{2 H}\right)+\sigma_{2}^{2}(\eta-t)+\lambda \sigma_{3}^{2}(\eta-t)}}^{\infty} \exp \left\{-\frac{1}{2}\right. \\
& \cdot\left(x+\sqrt{2 \sigma_{1}^{2}\left(\eta^{2 H}-t^{2 H}\right)+\sigma_{2}^{2}(\eta-t)+\lambda \sigma_{3}^{2}(\eta-t)}\right)^{2}+\left(r-q-\lambda \sigma_{3}\right)(\eta-t)+\sigma_{1}^{2}\left(\eta^{2 H}-t^{2 H}\right) \\
& \left.+\frac{\left(\sigma_{2}^{2}+\lambda \sigma_{3}^{2}\right)(\eta-t)}{2}\right\} \mathrm{d} x=\mathbb{b} \mathbb{M} r \int_{t}^{T} \exp \{-r(\eta-t)\} \sum_{n=0}^{\infty}\left\{\frac{\lambda^{n}(\eta-t)^{n} \exp [-\lambda(\eta-t)]}{n !} \mathscr{E}_{n}\right\} \cdot\{1 \\
& \left.-\mathbb{N}\left[\frac{\ln \left(\left(S \prod_{i=1}^{n}\left(1+j_{t_{i}}\right)\right) / S_{\eta}\right)+\left(r-q-\lambda \sigma_{3}\right)(\eta-t)-\sigma_{1}^{2}\left(\eta^{2 H}-t^{2 H}\right)-\left(\sigma_{2}^{2}+\lambda \sigma_{3}^{2}\right)(\eta-t) / 2}{2 \sigma_{1}^{2}\left(\eta^{2 H}-t^{2 H}\right)+\sigma_{2}^{2}(\eta-t)+\lambda \sigma_{3}^{2}(\eta-t)}\right]\right\} \mathrm{d} \eta-(q \\
& \left.+\lambda \sigma_{3}\right) S \int_{t}^{T} \exp \left\{-\left(q+\lambda \sigma_{3}\right)(\eta-t)\right\} \sum_{n=0}^{\infty}\left\{\frac{\lambda^{n}(\eta-t)^{n} \exp [-\lambda(\eta-t)]}{n !} \mathscr{E}_{n}\right\} \cdot\{1 \\
& \left.-\mathbb{N}\left[\frac{\ln \left(\left(S \prod_{i=1}^{n}\left(1+j_{t_{i}}\right)\right) / S_{\eta}\right)+\left(r-q-\lambda \sigma_{3}\right)(\eta-t)+\sigma_{1}^{2}\left(\eta^{2 H}-t^{2 H}\right)+\left(\sigma_{2}^{2}+\lambda \sigma_{3}^{2}\right)(\eta-t) / 2}{2 \sigma_{1}^{2}\left(\eta^{2 H}-t^{2 H}\right)+\sigma_{2}^{2}(\eta-t)+\lambda \sigma_{3}^{2}(\eta-t)}\right]\right\} \mathrm{d} \eta \text {. }
\end{aligned}
$$

Substituting (95) into (83), we have

$$
V\left(t, S_{t}, \mathbb{M}\right)=\mathbb{b} \mathbb{M}-S_{t}
$$

and then we obtain

$$
\mathbb{b M}-S_{t}=V_{E}\left(t, S_{t}, \mathbb{M}\right)+e\left(t, S_{t}, \mathbb{M}\right),
$$

$$
\begin{aligned}
S_{t} & =\mathbb{b} \mathbb{M}+S \exp \left\{-\left(q+\lambda \sigma_{3}\right)(T-t)\right\} \cdot \mathbb{N}\left\{-\left[-\ln \frac{S \prod_{i=1}^{n}\left(1+j_{t_{i}}\right)}{\mathfrak{b M} M}+\left(r-q-\lambda \sigma_{3}\right)(T-t)+\sigma_{1}^{2}\left(T^{2 H}-t^{2 H}\right)+\frac{1}{2}\left(\sigma_{2}^{2}\right.\right.\right. \\
& \left.\left.\left.+\lambda \sigma_{3}^{2}\right)(T-t)\right] \cdot\left[\sqrt{2 \sigma_{1}^{2}\left(T^{2 H}-t^{2 H}\right)+\sigma_{2}^{2}(T-t)+\lambda \sigma_{3}^{2}(T-t)}\right]^{-1}\right\}-\mathbb{b} \mathbb{M} \exp \{-r(T-t)\} \\
& \cdot \sum_{n=0}^{\infty}\left\{\frac{\lambda^{n}(T-t)^{n} \exp [-\lambda(T-t)]}{n !} \mathscr{E}_{n}\right\} \cdot \mathbb{N}\left\{-\left[-\ln \frac{S \prod_{i=1}^{n}\left(1+j_{t_{i}}\right)}{\mathfrak{b M} \mathbb{M}}+\left(r-q-\lambda \sigma_{3}\right)(T-t)-\sigma_{1}^{2}\left(T^{2 H}-t^{2 H}\right)\right.\right. \\
& \left.\left.-\frac{1}{2}\left(\sigma_{2}^{2}+\lambda \sigma_{3}^{2}\right)(T-t)\right] \cdot\left[\sqrt{2 \sigma_{1}^{2}\left(T^{2 H}-t^{2 H}\right)+\sigma_{2}^{2}(T-t)+\lambda \sigma_{3}^{2}(T-t)}\right]^{-1}\right\}-\mathbb{b M r} \int_{t}^{T} \exp \{-r(\eta-t)\}
\end{aligned}
$$




$$
\begin{aligned}
& \sum_{n=0}^{\infty}\left\{\frac{\lambda^{n}(T-t)^{n} \exp [-\lambda(T-t)]}{n !} \mathscr{E}_{n}\right\} \cdot\{1 \\
& -\mathbb{N}\left[\left(\ln \frac{S \prod_{i=1}^{n}\left(1+j_{t_{i}}\right)}{S_{\eta}}+\left(r-q-\lambda \sigma_{3}\right)(\eta-t)-\sigma_{1}^{2}\left(\eta^{2 H}-t^{2 H}\right)-\frac{1}{2}\left(\sigma_{2}^{2}+\lambda \sigma_{3}^{2}\right)(\eta-t)\right)\right. \\
& \left.\left.\cdot\left(\sqrt{2 \sigma_{1}^{2}\left(\eta^{2 H}-t^{2 H}\right)+\sigma_{2}^{2}(\eta-t)+\lambda \sigma_{3}^{2}(\eta-t)}\right)^{-1}\right]\right\} \mathrm{d} \eta+\left(q+\lambda \sigma_{3}\right) S \int_{t}^{T} \exp \left\{-\left(q+\lambda \sigma_{3}\right)(\eta-t)\right\} \\
& \cdot \sum_{n=0}^{\infty}\left\{\frac{\lambda^{n}(T-t)^{n} \exp [-\lambda(T-t)]}{n !} \mathscr{E}_{n}\right\} \cdot\{1 \\
& -\mathbb{N}\left[\left(\ln \frac{S \prod_{i=1}^{n}\left(1+j_{t_{i}}\right)}{S_{\eta}}+\left(r-q-\lambda \sigma_{3}\right)(\eta-t)+\sigma_{1}^{2}\left(\eta^{2 H}-t^{2 H}\right)+\frac{1}{2}\left(\sigma_{2}^{2}+\lambda \sigma_{3}^{2}\right)(\eta-t)\right)\right. \\
& \left.\left.\cdot\left(\sqrt{2 \sigma_{1}^{2}\left(\eta^{2 H}-t^{2 H}\right)+\sigma_{2}^{2}(\eta-t)+\lambda \sigma_{3}^{2}(\eta-t)}\right)^{-1}\right]\right\} \mathrm{d} \eta \text {. }
\end{aligned}
$$

In practice, it is very difficult to calculate nonlinear integral in (92); we only show that this assumption holds in Volterra integral equation for the optimal exercise boundary; by the relation (92) and Theorem 17, asymptotic expression of optimal exercise boundary $S=S_{t}$ is obtained near the maturity $t=T$. Comparing with Theorem 17 and Corollary 20, the optimal exercise boundary and Volterra integral equation of American fractional lookback call option are obvious as follows.

Corollary 21. Let $V^{*}(t, S$, m $)$ be American fractional lookback call option; then

$$
V^{*}(t, S, \text { mo })=V_{E}^{*}(t, S, \text { m })+e^{*}(t, S, \text { mo }),
$$

where $V_{E}^{*}(t, S, m)$ is price of the hedging portfolio of the equivalent European option, and

$$
\begin{aligned}
& V_{E}^{*}(t, S, \boldsymbol{m})=\sum_{n=0}^{\infty}\left\{\frac{\lambda^{n}(T-t)^{n} \exp [-\lambda(T-t)]}{n !} \mathscr{E}_{n}\right\} \\
& . {\left[S \prod_{i=1}^{n}\left(1+j_{t_{i}}\right) \exp \left\{-\left(q+\lambda \sigma_{3}\right)(T-t)\right\} \mathbb{N}\left(-\widehat{d}_{1}\right)\right.} \\
&\left.-\operatorname{amc} \exp \{-r(T-t)\} \mathbb{N}\left(-\widehat{d}_{2}\right)\right]
\end{aligned}
$$

where $\mathscr{E}_{n}$ denotes the expectation operator over the distribution of $\prod_{i=1}^{n}\left(1+j_{t_{i}}\right)$, खm is contractual strike price, $\mathbb{N}(\cdot)$ is the cumulative normal distribution function, and

$$
\begin{aligned}
\widehat{d}_{1} & =\left[\ln \frac{S \prod_{i=1}^{n}\left(1+j_{t_{i}}\right)}{\xi}+\left(r-q-\lambda \sigma_{3}\right)(T-t)\right. \\
& \left.+\sigma_{1}^{2}\left(T^{2 H}-t^{2 H}\right)+\frac{1}{2}\left(\sigma_{2}^{2}+\lambda \sigma_{3}^{2}\right)(T-t)\right]
\end{aligned}
$$

$$
\begin{aligned}
& \cdot\left(\sqrt{2 \sigma_{1}^{2}\left(T^{2 H}-t^{2 H}\right)+\sigma_{2}^{2}(T-t)+\lambda \sigma_{3}^{2}(T-t)}\right)^{-1}, \\
\widehat{d}_{2}= & \widehat{d}_{1} \\
& -\sqrt{2 \sigma_{1}^{2}\left(T^{2 H}-t^{2 H}\right)+\sigma_{2}^{2}(T-t)+\lambda \sigma_{3}^{2}(T-t)},
\end{aligned}
$$

$e^{*}\left(t, S, \mathrm{~m}^{\prime}\right)$ is early exercise premium, and its explicit integral can be represented by

$$
\begin{aligned}
& e^{*}(t, S, \mathbb{m}) \\
& \quad=\int_{t}^{T} \mathrm{~d} \eta \int_{0}^{S_{\eta}}\left[\left(q+\lambda \sigma_{3}\right) \xi-₫ \square r\right] \cdot \mathbb{G}(S, t ; \xi, \eta) \mathrm{d} \xi
\end{aligned}
$$

\section{Simulation Studies}

In the whole domain $\mathfrak{D}=\{(t, S, \mathbb{M}) \in[0, T] \times[\mathbb{M}, \infty) \times$ $\left.\mathbb{R}_{+}\right\}$of the American fractional floating strike lookback put option model, the American put is alive when $S<\mathbb{M}$ and becomes dead when $S>\mathbb{M}$. The boundary which divides the continuation region (option remains alive) and the stopping region (option becomes dead) is time dependent; that is, $S$ is a function of $t$. Similar to the usual argument for American floating strike lookback put options the critical exercise price should be denoted as [30]

$$
\underline{S}(t, \mathbb{M})=\frac{S_{t}(t, S, \mathbb{M})}{\mathbb{M}} .
$$

As deduced from (92), at a given time $t$, the optimal exercise boundary $S_{t}(t, S, \mathbb{M})$ increases linearly with $\mathbb{M}$. Since both $\underline{S}(t, \mathbb{M})$ and $\mathbb{M}$ are increasing function of time $t, S_{t}(t, S, \mathbb{M})$ increases as time is approaching expiration. 

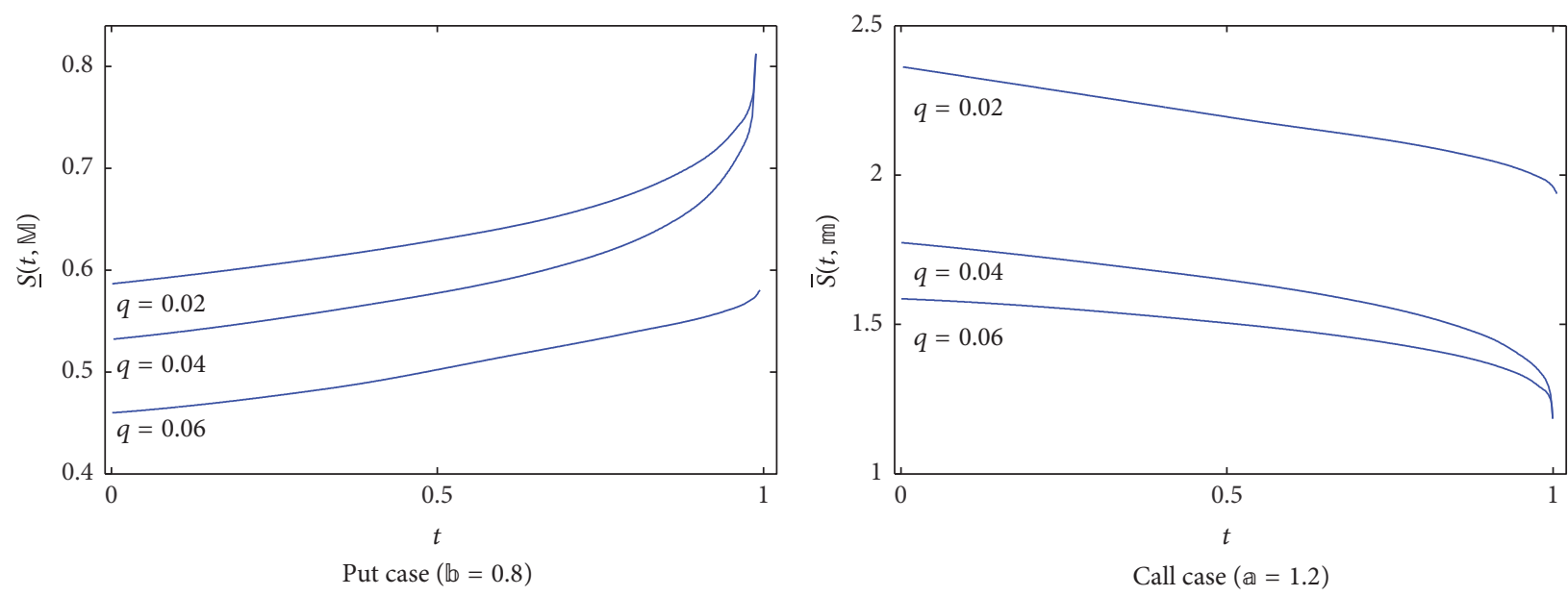

Figure 1: Plot of $\underline{S}(t, \mathbb{M})$ and $\bar{S}(t, m)$ against time $t$ for American fractional lookback options with varying $q=0.02,0.04,0.06$.

Similarly, the critical exercise price for the American fractional floating strike lookback call option should be denoted as

$$
\bar{S}(t, m)=\frac{S_{t}(t, S, m)}{m},
$$

where $\bar{S}(t, m)$ is a monotonically decreasing function of time $t$. The plots of $\underline{S}(t, \mathbb{M})$ and $\bar{S}(t, \mathrm{~m})$ against time $t$ with varying interest rate are shown in Figure 1 , where $\mathbb{m}=\mathbb{M}=100$, $T=1, r=0.05, q=0.02,0.04,0.06, \sigma_{1}=0.2, \sigma_{2}=0.3$, $\sigma_{3}=0.0071, \lambda=2.68, \mathbb{}=1.2$, and $\mathbb{b}=0.8$. Since lower value of interest rate leads to the loss of the time value of the floating strike price to be smaller when the American fractional floating strike lookback call option is exercised prematurely, then the critical exercise price decreases when the interest rate assumes lower value. The similar phenomenon also appeared in [30].

The limiting behaviors at times close to maturity of the critical exercise prices for the American fractional floating strike lookback put and call options are, respectively, given by

$$
\begin{aligned}
& \lim _{t \rightarrow T^{-}} S_{t}(t, S, \mathbb{M})=\frac{1}{\mathbb{a}} \min \left(\frac{r}{q+\lambda \sigma_{3}}, 1\right) \lim _{t \rightarrow T^{-}} \mathbb{M}_{t}, \\
& \lim _{t \rightarrow T^{-}} S_{t}(t, S, \mathbb{m})=\frac{1}{\mathfrak{b}} \max \left(\frac{r}{q+\lambda \sigma_{3}}, 1\right) \lim _{t \rightarrow T^{-}} m_{t} .
\end{aligned}
$$

Hence the strike prices of the American fractional lookback put and call options are set to be $\mathbb{M}_{0}^{T}$ and $\mathrm{mo}_{0}^{T}$, respectively. The usual argument and proof of analysis of limiting behaviors can be applied in a similar manner like literature $[28,30]$.

With respect to the early exercise premium of the American fractional lookback option, we intuitively suppose that it strongly depends on the sign of the drift coefficient of the price process $\left(S_{t}\right)_{t \geq 0}$. If $r-q-\lambda \sigma_{3}<0$, then $S_{t}$ decreases to $\mathrm{mon}_{T}$ as $t \rightarrow T$ in an average sense, so that $\left(S_{t}-\mathrm{amm}_{t}\right)^{+} \rightarrow 0$ and $\left(\mathbb{b} M_{t}-S_{t}\right)^{+}$grows as $t \rightarrow T$ in the same sense. This implies that early exercise before maturity is optimal for call but not for put, at least in the average sense. On the contrary, if $r-q-\lambda \sigma_{3}>0$, then the reverse would be true. For put options with $r-q-\lambda \sigma_{3}<0$ and call options with $r-q-\lambda \sigma_{3}>0$, the early exercise premiums should be negligible, and hence there should be almost no difference between the values of American and European options. To confirm this expectation, we compare the values of American and European options with $r-q-\lambda \sigma_{3}<0$ in Figure 2 for call and put case, where American (European) values are drawn in blue (red) lines. Further numerical experiments confirmed that the same expectation is realized for the critical case $r-$ $q-\lambda \sigma_{3}=0$, too. Also, we observed from these figures that the call (put) values are monotonically decreasing (increasing) in Figure 2, the option values are convex functions of $S$, and the option values are relatively insensitive to the asset price $S$ when the option is out-of-the-money. Notice that the call (put) values are almost increasing (decreasing) in $S$ when the option is in-the-money but not when $S=\mathbb{M}$ and $b=1$. From other numerical experiments, we can conclude that the adjustable constants $₫ \geq 2$ and $\mathbb{b} \leq 0.5$ are practically insignificant, because the option values are negligible for those cases.

\section{Conclusions}

In this paper, we compound the Brownian motion, fraction Brownian motion, and Poisson process by fractional Wick-Itô-Skorohod integral. In our new pricing model, the fundamental solutions of stochastic parabolic partial differential equations are estimated under the condition of Merton assumptions. By solving the free boundary problem and the Black-Scholes-Merton partial differential equations, we characterized asymptotic behaviors of the early exercise boundaries at a time close to expiration and at infinite time to expiration. Since the fundamental solution of stochastic partial differential equations plays an important role in numerical inversion for the put and call case, then we characterized asymptotic behaviors of the early exercise boundaries at a time close to expiration and at infinite time to expiration. The numerical simulation reveals some notable features of the fractional lookback options: (1) For call (put) options 

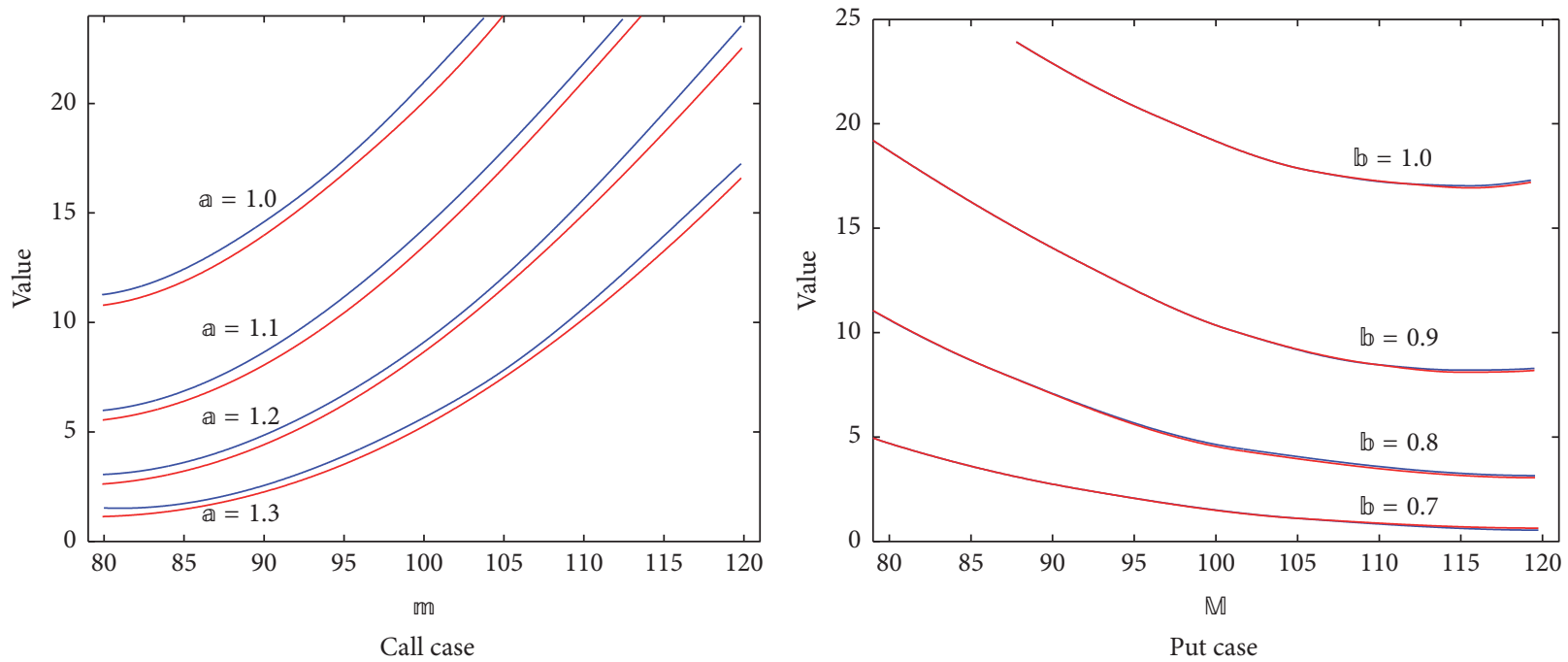

Figure 2: Values of American and European fractional lookback options $\left(t=0, T=1\right.$, m $=80, \mathbb{M}=120, r-q-\lambda \sigma_{3}=0.05, \AA=1.0,1.1,1.2,1.3$, $\mathfrak{b}=0.7,0.8,0.9,1.0)$.

with $r \geq q+\lambda \sigma_{3}\left(r \leq q+\lambda \sigma_{3}\right)$, there is little difference in value between American and European options. This readily suggests that investors should buy American options, because the holder of American options has a benefit of clearing his investment position at any time, even if he unexpectedly encounters the opportunity of terminating the contract. The early exercise boundary is of practical value for making decisions by the option holders. (2) The fractional lookback scheme with the adjustable constants $₫ \geq 1$ and $b \leq 1$ is extremely effective for reducing option premiums, especially for low volatile cases. With $\mathbb{a}=1.2$ and $\mathbb{b}=0.8$, we can achieve larger reductions of the option premiums from the standard cases with $\mathbb{a}=\mathbb{b}=1$. The fractional lookback options must be more attractive to investors than the standard floating strike options.

\section{Conflicts of Interest}

The author declares that there are no conflicts of interest regarding the publication of this paper.

\section{Acknowledgments}

This work was partially supported by the Young Teacher Research Project of Lanzhou University of Finance and Economics (Lzufe2017) and the Scientific Research Foundation of Gansu Provincial Education Department (Grant no. 3ZS042-B25-049).

\section{References}

[1] M. Zili, "On the mixed fractional Brownian motion," Journal of Applied Mathematics and Stochastic Analysis. JAMSA, Article ID 32435, Art. ID 32435, 9 pages, 2006.

[2] P. Cheridito, "Mixed fractional Brownian motion," Bernoulli. Official Journal of the Bernoulli Society for Mathematical Statistics and Probability, vol. 7, no. 6, pp. 913-934, 2001.
[3] W.-L. Xiao, W.-G. Zhang, X. Zhang, and X. Zhang, "Pricing model for equity warrants in a mixed fractional Brownian environment and its algorithm," Physica A. Statistical Mechanics and its Applications, vol. 391, no. 24, pp. 6418-6431, 2012.

[4] C. El-Nouty, "'The fractional mixed fractional Brownian motion," Statistics Probability and Letters, vol. 65, no. 2, pp. 111120, 2003.

[5] L. Sun, "Pricing currency options in the mixed fractional Brownian motion," Physica A. Statistical Mechanics and its Applications, vol. 392, no. 16, pp. 3441-3458, 2013.

[6] Y. Mishura and E. Valkeila, "The absence of arbitrage in a mixed Brownian-fractional Brownian model," in Proceedings of the Steklov Institute of Mathematics, vol. 237, pp. 224-233, 2002.

[7] X. He and W. Chen, "The pricing of credit default swaps under a generalized mixed fractional Brownian motion," Physica A. Statistical Mechanics and its Applications, vol. 404, pp. 26-33, 2014.

[8] X.-T. Wang, E.-H. Zhu, M.-M. Tang, and H.-G. Yan, "Scaling and long-range dependence in option pricing II: pricing European option with transaction costs under the mixed BROwnian-fractional BROwnian model," Physica A. Statistical Mechanics and its Applications, vol. 389, no. 3, pp. 445-451, 2010.

[9] X.-C. Sun and L.-T. Yan, "Mixed-fractional models to credit risk pricing," Journal of Statistical and Econometric Methods, vol. 1, no. 3, pp. 79-96, 2012.

[10] F. Biagini, Y.-Z. Hu, B. Øksendal, and T.-S. Zhang, Stochastic Calculus for Fractional Brownian Motion and Applications, Springer-Verlag, London, UK, 2008.

[11] B. Tomas and H. Henrik, "A note on Wick products and the fractional Black-Scholes model," Finance and Stochastics, vol. 9, no. 2, pp. 197-209, 2005.

[12] Y.-Z. Hu and B. Øksendal, "Fractional white noise calculus and applications to finance, Infinite Dimensional Analysis," Quantum Probability and Related Topics, vol. 6, no. 1, p. 32, 2003.

[13] M.-A. Chang, C. Chinhyung, and P. Keenwan, "The price of foreign currency options under jump-diffusion processes," Journal of Futures Markets, vol. 27, no. 7, pp. 669-695, 2007. 
[14] W.-L. Xiao, W.-G. Zhang, X.-L. Zhang, and Y.-L. Wang, "Pricing currency options in a fractional Brownian motion with jumps," Economic Modelling, vol. 27, no. 5, pp. 935-942, 2010.

[15] Á. Cartea and D. del-Castillo-Negrete, "Fractional diffusion models of option prices in markets with jumps," Physica A: Statistical Mechanics and Its Applications, vol. 374, no. 2, pp. 749-763, 2007.

[16] C. Rama and T. Peter, "Non-parametric calibration of jumpdiffusion option pricing models," Journal of Computational Finance, vol. 7, no. 3, pp. 1-49, 2004.

[17] S. Beckers, "A note on estimating the parameters of the diffusion-jump model of stock returns," Journal of Financial and Quantitative Analysis, vol. 16, no. 1, pp. 127-140, 1981.

[18] S. G. Kou, "A jump-diffusion model for option pricing," Management Science, vol. 48, no. 8, pp. 1086-1101, 2002.

[19] B. L. Prakasa rao, "Option pricing for processes driven by mixed fractional Brownian motion with superimposed jumps," Probability in the Engineering and Informational Sciences, vol. 29, no. 4, pp. 589-596, 2015.

[20] F. Shokrollahi and A. Kilicman, "Pricing currency option in a mixed fractional Brownian motion with jumps environment," Mathematical Problems in Engineering, vol. 2014, no. 1, Article ID 858210, 13 pages, 2014.

[21] F. Shokrollahi and A. Kilicman, "Actuarial approach in a mixed fractional Brownian motion with jumps environment for pricing currency option," Advances in Difference Equations, vol. 2015, no. 1, p. 1, 2015.

[22] L.-M. Feng and V. Linetsky, "Computing exponential moments of the discrete maximum of a Lévy process and lookback options," Finance and Stochastics, vol. 13, no. 4, pp. 501-529, 2009.

[23] A. Conze and R. Viswanathan, "Path dependent options, the case of lookback options," The Journal of Finance, vol. 46, no. 5, pp. 1893-1907, 1991.

[24] K. S. Leung, "An analytic pricing formula for lookback options under stochastic volatility," Applied Mathematics Letters. An International Journal of Rapid Publication, vol. 26, no. 1, pp. 145149, 2013.

[25] S.-H. Park and J.-H. Kim, "An semi-analytic pricing formula for lookback options under a general stochastic volatility model," Statistics and Probability Letters, vol. 83, no. 11, pp. 2537-2543, 2013.

[26] C.-D. Fuh, S.-F. Luo, and J.-F. Yen, "Pricing discrete pathdependent options under a double exponential jump-diffusion model," Journal of Banking and Finance, vol. 37, no. 8, pp. 27022713, 2013.

[27] E. Eberlein and A. Papapantoleon, "Equivalence of floating and fixed strike Asian and lookback options," Stochastic Processes and their Applications, vol. 115, no. 1, pp. 31-40, 2005.

[28] T. L. Lai and T. W. Lim, "Exercise regions and efficient valuation of American lookback options," Mathematical Finance. An International Journal of Mathematics, Statistics and Financial Economics, vol. 14, no. 2, pp. 249-269, 2004.

[29] C. Necula, "Option pricing in a fractional Brownian motion environment," Math. Reports(Bucur.), vol. 6, no. 3, pp. 259-273, 2004.

[30] H. Yu, Y. K. Kwok, and L. Wu, "Early exercise policies of American floating strike and fixed strike lookback options," vol. 47, no. 7, pp. 4591-4602. 


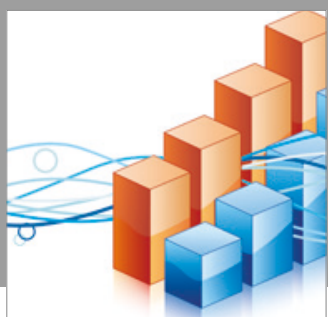

Advances in

Operations Research

vatersals

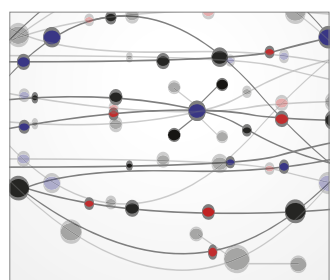

\section{The Scientific} World Journal
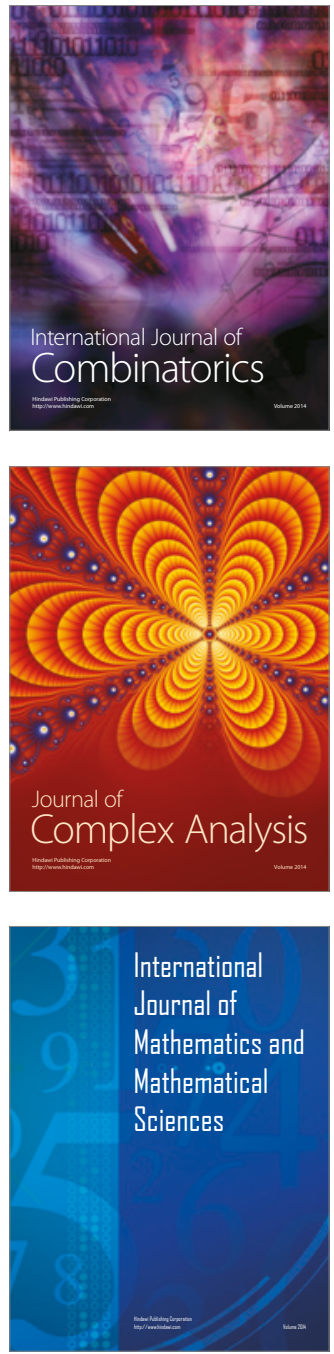
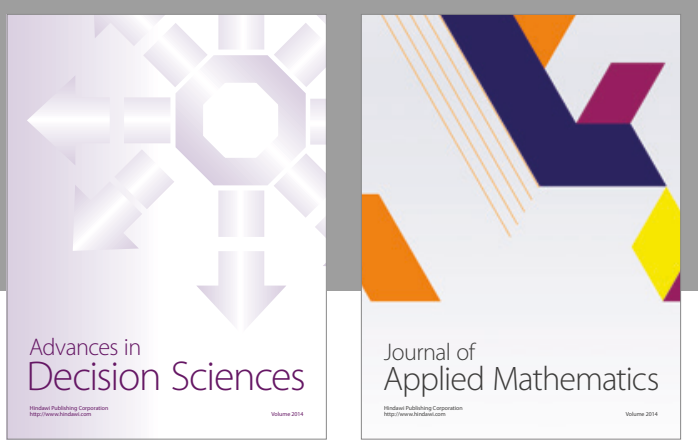

Algebra

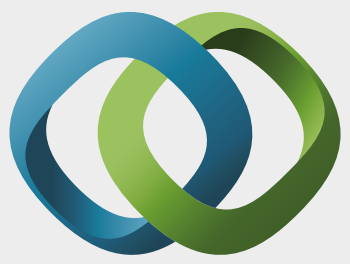

\section{Hindawi}

Submit your manuscripts at

https://www.hindawi.com
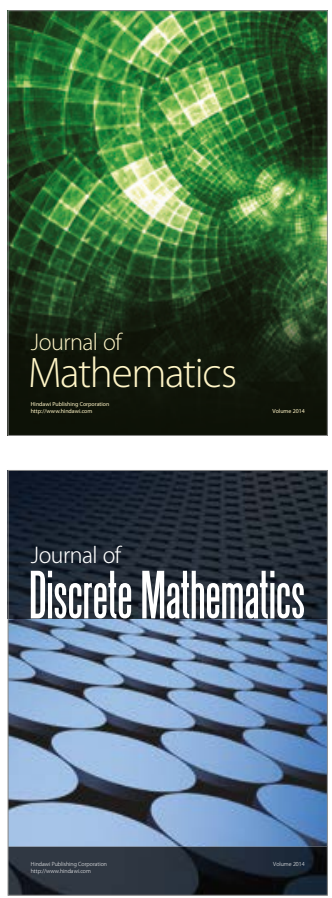

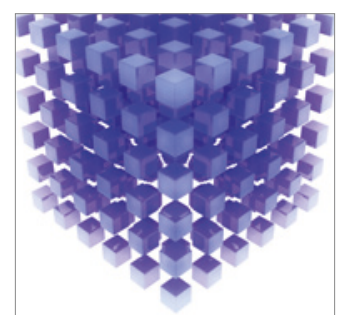

Mathematical Problems in Engineering
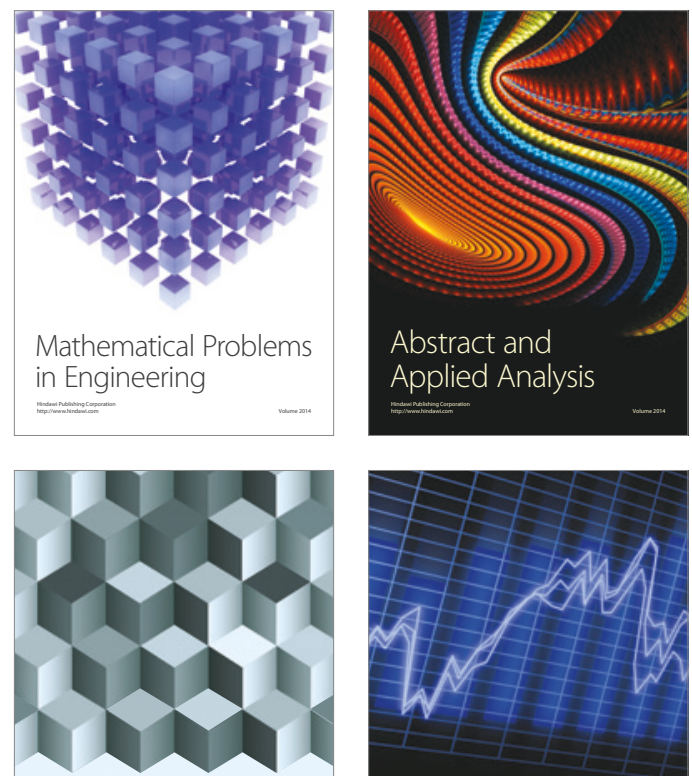

Journal of

Function Spaces

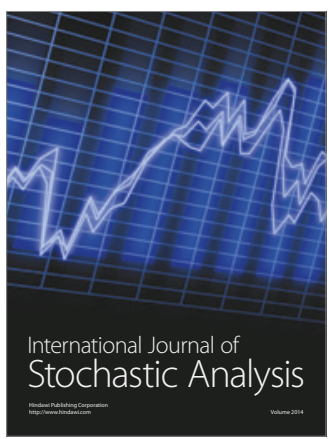

Probability and Statistics
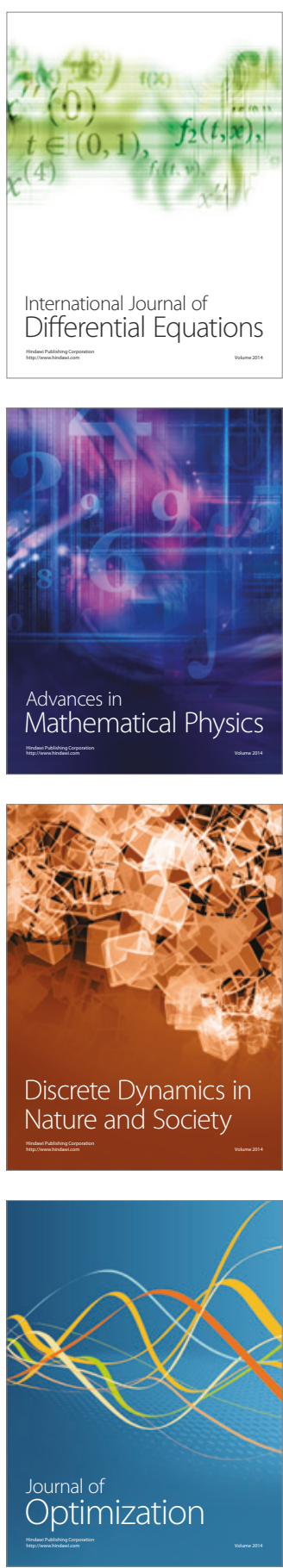